\title{
Exponential Stability of Stochastic Delayed Neural Networks with Inverse Hölder Activation Functions and Markovian Jump Parameters
}

\author{
Yingwei $\mathrm{Li}^{1}$ and Huaiqin $\mathrm{Wu}^{2}$ \\ ${ }^{1}$ College of Information Science and Engineering, Yanshan University, Qinhuangdao 066004, China \\ ${ }^{2}$ College of Science, Yanshan University, Qinhuangdao 066001, China
}

Correspondence should be addressed to Huaiqin Wu; huaiqinwu@ysu.edu.cn

Received 21 September 2013; Accepted 15 December 2013; Published 23 January 2014

Academic Editor: Sarangapani Jagannathan

Copyright (C) 2014 Y. Li and H. Wu. This is an open access article distributed under the Creative Commons Attribution License, which permits unrestricted use, distribution, and reproduction in any medium, provided the original work is properly cited.

\begin{abstract}
The exponential stability issue for a class of stochastic neural networks (SNNs) with Markovian jump parameters, mixed time delays, and $\alpha$-inverse Hölder activation functions is investigated. The jumping parameters are modeled as a continuous-time finite-state Markov chain. Firstly, based on Brouwer degree properties, the existence and uniqueness of the equilibrium point for SNNs without noise perturbations are proved. Secondly, by applying the Lyapunov-Krasovskii functional approach, stochastic analysis theory, and linear matrix inequality (LMI) technique, new delay-dependent sufficient criteria are achieved in terms of LMIs to ensure the SNNs with noise perturbations to be globally exponentially stable in the mean square. Finally, two simulation examples are provided to demonstrate the validity of the theoretical results.
\end{abstract}

\section{Introduction}

In the past few decades, there has been increasing interest in different classes of neural networks such as Hopfield, cellular, Cohen-Grossberg, and bidirectional associative neural networks due to their potential applications in many areas such as classification, signal and image processing, parallel computing, associate memories, optimization, and cryptography [1-6]. In the design of practical neural networks, the qualitative analysis of neural network dynamics plays an important role. To solve problems of optimization, neural control, signal processing, and so forth, neural networks have to be designed in such a way that, for a given external input, they exhibit only one globally asymptotically/exponentially stable equilibrium point. Hence, much effort has been made in the stability of neural networks, and a number of sufficient conditions have been proposed to guarantee the global asymptotic/exponential stability for neural networks with or without delays; see, for example, [7-19] and the references therein.

As is well known, a real system is usually affected by external perturbations which in many cases are of great uncertainty and hence may be treated as random. As pointed out in [20], in real nervous systems, and in the implementation of artificial neural networks, synaptic transmission is a noisy process brought on by random fluctuations from the release of neurotransmitters and other probabilistic causes; hence noise is unavoidable and should be taken into consideration in modeling. Moreover, in $[21,22]$, it has been shown that a neural network can be stabilized or destabilized by certain stochastic inputs. Therefore, the stochastic stability of various neural networks with or without delays under noise disturbance has received extensive attention from a lot of scholars in recent years, and some results related to this issue have been reported in the literature; see [23-31].

When the neural network incorporates abrupt changes in its structure, the Markovian jumping (switching) nonlinear system is very appropriate to describe its dynamics. In the past few years, based on Markovian switching system theory, the dynamics of various neural networks with Markovian jump parameters have been widely explored in the existing literature. In [32], Markovian jumping BAM neural networks with time-varying delays were investigated, and some 
sufficient conditions were derived for the global exponential stability in the mean square by using stochastic LyapunovKrasovskii functional approach. In [33], uncertain Markovian jumping Cohen-Grossberg neural networks with mixed time-varying delays were discussed, and the robust stability results were obtained in terms of LMIs. In [34], the authors considered the robust stabilization of stochastic Markovian jumping dynamical networks with mode-dependent mixed delays. In [35, 36], Markovian jumping recurrent neural networks with discrete and distributed delays and with interval time-varying delays were investigated, respectively, and some criteria had been established to guarantee the existence of the state estimators. In [37], Markovian coupled neural networks with nonidentical node-delays and random coupling strengths were introduced, and several delaydependent sufficient synchronization criteria ware derived and formulated by LMIs. Very recently, considerable efforts have been devoted to investigate the Markovian jumping SNNs; various stability conditions have been presented in the existing literature. In [38], the global stability issue for Markovian jumping stochastic Cohen-Grossberg neural networks with mixed time delays was studied, and the exponential stability results were proposed by using stochastic LyapunovKrasovskii functional approach. In [39], a class of SNNs with both Markovian jump parameters and mixed time delays was investigated, and some novel sufficient conditions which guarantee the exponential stability of the equilibrium point in the mean square were derived in terms of LMIs. Furthermore, the robust exponential stability was discussed for a class of Markovian jump impulsive stochastic CohenGrossberg neural networks with mixed time delays by using inequality techniques and Lyapunov method in [40]. In [41], some sufficient conditions were presented in terms of LMIs to guarantee the global exponential stability for stochastic jumping BAM neural networks with time-varying and distributed delays. In [42], delay-interval-dependent robust stability results were addressed in terms of LMIs for uncertain stochastic systems with Markovian jumping parameters. In [42], the stochastic global exponential stability problem was considered, and some delay-dependent exponential stability criteria and decay estimation are presented in terms of LMIs for neutral-type impulsive neural networks with mixed timedelays and Markovian jumping parameters.

It should be noted that all the results reported in the literature above are concerned with Markovian jumping SNNs with Lipschitz neuron activation functions. To the best of our knowledge, up to now, very little attention has been paid to the problem of the global exponential stability of Markovian jumping SNNs with non-Lipschitz activation functions, which often appear in realistic neural networks. This situation motivates our present investigation.

In this paper, our aim is to study the delay-dependent exponential stability problem for a class of Markovian jumping neural networks with mixed time delays and $\alpha$-inverse Hölder activation functions under stochastic noise perturbation. Here, it should be pointed out that $\alpha$-inverse Hölder activation functions are a class of non-Lipschitz functions. By utilizing the Brouwer degree properties, Lyapunov stability theory, stochastic analysis theory, and LMI technique, some novel delay-dependent conditions are obtained, which guarantee the exponential stability of the equilibrium point. The results obtained in this paper improve and generalize those presented in $[14,15,17-19]$ since our model and conditions are more general and weaker than those presented in [14, $15,17-19]$, whereas the criteria obtained in $[14,15]$ were not expressed in terms of LMIs, and the noise disturbance was not considered in [14, 15, 17-19].

The rest of this paper is organized as follows. In Section 2, the model of SNNs with both mixed time delays and inverse Hölder activation functions is introduced, together with some definitions and lemmas. By means of topological degree theory and Lyapunov-Krasovskii functional approach, our main results are established in Section 3. In Section 4, two numerical examples are presented to show the effectiveness of the obtained results. Finally, some conclusions are given in Section 5 .

Notations. Throughout this paper, $R$ denotes the set of real numbers, $R^{n}$ denotes the n-dimensional Euclidean space, and $R^{m \times n}$ denotes the set of all $m \times n$ real matrices. For any matrix $A, A^{T}$ denotes the transpose of $A$ and $A^{-1}$ denotes the inverse of $A$. If $A$ is a real symmetric matrix, $A>0(A<0)$ means that $A$ is positive definite (negative definite). $\lambda_{\min }(\cdot)$ and $\lambda_{\max }(\cdot)$ denote net minimum and maximum eigenvalues of a real symmetric matrix, respectively. $I_{n}$ is the $n \times n$ identity matrix; the notation $\mathscr{C}^{2.1}\left(R^{+} \times R^{n} \times S ; R^{+}\right)$denotes the family of all nonnegative functions $V(t, u(t), i)$ on $R^{+} \times R^{n} \times S$ which are continuously twice differentiable in $u$ and once differentiable in $t ;(\mathbb{A}, \mathscr{F}, \mathscr{P})$ is a complete probability space, where $\mathbb{A}$ is the sample space, $\mathscr{F}$ is the $\sigma$-algebra of subsets of the sample space, and $\mathscr{P}$ is the probability measure on $\mathscr{F} ; L_{\mathscr{F}_{0}}^{2}\left([-\omega, 0] ; R^{n}\right)$ denotes the family of all $\mathscr{F}_{0}$-measurable $C\left([-\omega, 0] ; R^{n}\right)$-valued random variables $\xi=\{\xi(\theta):-\omega \leq$ $\theta \leq 0\}$ such that $\sup _{-\omega \leq \theta \leq 0} \mathbb{E}|\xi(\theta)|<\infty$, where $\mathbb{E}\{\cdot\}$ stands for the mathematical expectation operator with respect to the given probability measure $\mathscr{P}$. Given the column vectors $x=\left(x_{1}, \ldots, x_{n}\right)^{T}, y=\left(y_{1}, \ldots, y_{n}\right)^{T}, x^{T} y=\sum_{i=1}^{n} x_{i} y_{i}$, $\|x\|=\left(\sum_{i=1}^{n} x_{i}^{2}\right)^{1 / 2} \cdot \dot{x}(t)$ denotes the derivative of $x(t)$, and $*$ represents the symmetric form of matrix.

\section{Model Description and Preliminaries}

In this paper, the stochastic neural networks with mixed time delays are described by the following integrodifferential equation system:

$$
\begin{aligned}
d x(t)= & {\left[-C x(t)+W_{0} f(x(t))+W_{1} g(x(t-\tau(t)))\right.} \\
& \left.+W_{2} \int_{t-\sigma}^{t} h(x(s)) d s+J\right] d t \\
& +\rho(x(t), x(t-\tau(t), t)) d \omega(t),
\end{aligned}
$$

where $x(t)=\left(x_{1}(t), \ldots, x_{n}(t)\right)^{T}$ denotes the state at time $t$; $f(\cdot), g(\cdot)$, and $h(\cdot)$ denote the neuron activation, and $f(x(t))=\left(f_{1}\left(x_{1}(t)\right), \ldots, f_{n}\left(x_{n}(t)\right)\right)^{T}, g(x(t))=\left(g_{1}\left(x_{1}(t)\right)\right.$, 
$\left.\ldots, g_{n}\left(x_{n}(t)\right)\right)^{T}$, and $h(x(t))=\left(h_{1}\left(x_{1}(t)\right), \ldots, h_{n}\left(x_{n}(t)\right)\right)^{T}$; $C=\operatorname{diag}\left\{c_{i}\right\}$ is a positive diagonal matrix; $c_{i}>0, i=$ $1, \ldots, n$, are the neural self-inhibitions; $W_{0}=\left(W_{0 i j}\right)_{n \times n}, W_{1}=$ $\left(W_{1 i j}\right)_{n \times n}$, and $W_{2}=\left(W_{2 i j}\right)_{n \times n}$ denote the connection weight matrix, the discretely delayed connection weight matrix, and the distributively delayed connection weight matrix, respectively; $J=\left(J_{1}, \ldots, J_{n}\right)$ is the external input; $\tau(t)$ is the discrete time-varying delay which is bounded with $0<$ $\tau(t)<\bar{\tau}$ and $\dot{\tau}(t) \leq \tau<1 ; \sigma$ is a constant delay; $\rho$ : $R^{n} \times R^{n} \times R^{+} \rightarrow R^{n}$ denotes the stochastic disturbance; $\omega(t)=\left(\omega_{1}(t), \ldots, \omega_{n}(t)\right)^{T}$ is $n$-dimensional Brownian motion defined on a complete probability space $(\mathbb{A}, \mathscr{F}, P)$ with a filtration $\left\{\mathscr{F}_{t}\right\}_{t \geq 0}$ satisfying the usual conditions (i.e., it is right continuous and $\mathscr{F}_{0}$ contains all $P$-null sets) and

$$
\mathbb{E}\{d \omega(t)\}=0, \quad \mathbb{E}\left\{d \omega^{2}(t)\right\}=d t .
$$

Suppose that the initial condition of the neural network (1) has the form $x(t)=\varphi(t)$ for $t \in[-\Phi, 0]$, where $\varphi(t)$ are continuous functions; $\omega=\max (\bar{\tau}, \sigma)$.

Throughout this paper, we assume that the activation functions $f_{i}, g_{i}$, and $h_{i}, i=1,2, \ldots, n$, satisfy the following.

$\left(H_{1}\right)$ We have the following.

(1) $f_{i}$ is a monotonic increasing continuous function.

(2) For any $\rho \in R$, there exist the scalars $q_{\rho}>0$, $r_{\rho}>0$ which are correlated with $\rho$, and $\alpha>0$ such that

$$
\left|f_{i}(\theta)-f_{i}(\rho)\right| \geq q_{i_{\rho}}|\theta-\rho|^{\alpha}, \quad \forall|\theta-\rho| \leq r_{i_{\rho}} .
$$

$\left(H_{2}\right) g_{i}, h_{i}$ are continuous, and $\left|g_{i}\left(s_{1}\right)-g_{i}\left(s_{2}\right)\right| \leq$ $e_{i}\left|f_{i}\left(s_{1}\right)-f_{i}\left(s_{2}\right)\right|,\left|h_{i}\left(s_{1}\right)-h_{i}\left(s_{2}\right)\right| \leq k_{i}\left|f_{i}\left(s_{1}\right)-f_{i}\left(s_{2}\right)\right|$, for all $s_{1}, s_{2} \in R, s_{1} \neq s_{2}, i=1,2, \ldots, n$. Denote $E=$ $\operatorname{diag}\left\{e_{i}\right\}, K=\operatorname{diag}\left\{k_{i}\right\}$.

Remark 1. In [15], the function $f_{i}$ satisfying assumption $\left(H_{1}\right)$ is said to be an $\alpha$-inverse Hölder function and firstly is used as the neuron activation function in the study of the stability issue study of neural networks. It is easy to check that $\alpha$-inverse Hölder functions are a class of nonLipschitz functions, and there exist a great number of $\alpha$ inverse Hölder functions in the engineering mathematics; for example, $f(\theta)=\arctan \theta$ and $f(\theta)=\theta^{3}+\theta$ are 1-inverse Hölder functions; $f(\theta)=\theta^{3}$ is a 3 -inverse Hölder function.

Remark 2. From $\left(H_{2}\right)$, we can get that $e_{i}$ and $k_{i}$ are positive scalars, so $E$ and $F$ are both positive definite diagonal matrices.

Remark 3. The relations among the different activation functions $f_{i}$ (which are $\alpha$-inverse Hölder activation functions), $g_{i}$, and $h_{i}$ are implicitly established in Theorem 14 . Such relations however have not been provided in other reported literature.
For the deterministic neural network system

$$
\begin{gathered}
d x(t)=\left[-C x(t)+W_{0} f(x(t))+W_{1} g(x(t-\tau(t)))\right. \\
\left.+W_{2} \int_{t-\sigma}^{t} h(x(s)) d s+J\right] d t .
\end{gathered}
$$

We have the following result.

Theorem 4. Under assumptions $\left(H_{1}\right)$ and $\left(H_{2}\right)$, if there exist a positive definite diagonal matrix $P$ and two positive definite matrices $S, T$ such that the following condition is satisfied:

$$
\left(\begin{array}{ccc}
P W_{0}+W_{0}^{T} P+E^{2} S+\sigma K^{2} T & P W_{1} & P W_{2} \\
W_{1}^{T} P & -S & 0 \\
W_{2}^{T} P & 0 & -\sigma^{-1} T
\end{array}\right)<0,
$$

then the neural network system (4) has a unique equilibrium point.

Proof. See the appendix.

In order to guarantee that system (1) has equilibrium, we assume that as the system approaches to its equilibrium, the stochastic noise contribution vanishes; that is,

$$
\left(H_{3}\right) \rho\left(x^{*}, x^{*}, t\right)=0, i=1,2, \ldots, n .
$$

Thus, the system (1) admits one equilibrium point $x^{*}=$ $\left(x_{1}^{*}, x_{2}^{*}, \ldots, x_{n}^{*}\right)^{T}$ under $\left(H_{3}\right)$. Let $u(t)=x(t)-x^{*} ;$ the system (1) can be rewritten in the following form:

$$
\begin{aligned}
d u(t)= & {\left[-C u(t)+W_{0} \bar{f}(u(t))+W_{1} \bar{g}(u(t-\tau(t)))\right.} \\
& \left.+W_{2} \int_{t-\sigma}^{t} \bar{h}(u(s)) d s\right] d t \\
& +\sigma(u(t), u(t-\tau(t), t)) d \omega(t)
\end{aligned}
$$

where

$$
\begin{gathered}
u(t)=\left(u_{1}(t), u_{2}(t), \ldots, u_{n}(t)\right)^{T}, \\
\bar{f}(u(t))=\left(\bar{f}_{1}\left(u_{1}(t)\right), \bar{f}_{2}\left(u_{2}(t)\right), \ldots, \bar{f}_{n}\left(u_{n}(t)\right)\right)^{T}, \\
\bar{g}(u(t-\tau(t)))=\left(\bar{g}_{1}\left(u_{1}(t-\tau(t))\right), \bar{g}_{2}\left(u_{2}(t-\tau(t))\right),\right. \\
\left.\ldots, \bar{g}_{n}\left(u_{n}(t-\tau(t))\right)\right)^{T}, \\
\bar{h}(u(t))=\left(\bar{h}_{1}\left(u_{1}(t)\right), \bar{h}_{2}\left(u_{2}(t)\right), \ldots, \bar{h}_{n}\left(u_{n}(t)\right)\right)^{T}, \\
\bar{f}_{i}\left(u_{i}(t)\right)=f_{i}\left(u_{i}(t)+x_{i}^{*}\right)-f_{i}\left(x_{i}^{*}\right), \\
\bar{g}_{i}\left(u_{i}(t-\tau(t))\right)=g_{i}\left(u_{i}(t-\tau(t))+x_{i}^{*}\right)-g_{i}\left(x_{i}^{*}\right), \\
\bar{h}_{i}\left(u_{i}(t)\right)=h_{i}\left(u_{i}(t)+x_{i}^{*}\right)-h_{i}\left(x_{i}^{*}\right), \\
\sigma(u(t), u(t-\tau(t)), t)=\rho\left(u(t)+x^{*}, u(t-\tau(t))+x^{*}, t\right),
\end{gathered}
$$




$$
\begin{aligned}
& \sigma(u(t), u(t-\tau(t)), t) \\
& =\left(\sigma_{1}\left(u_{1}(t), u_{1}(t-\tau(t)), t\right),\right. \\
& \left.\quad \ldots, \sigma_{n}\left(u_{n}(t), u_{n}(t-\tau(t)), t\right)\right)^{T} .
\end{aligned}
$$

Apparently, $\bar{f}_{i}(s)$ is also an $\alpha$-inverse Hölder function, and $\bar{f}_{i}(0)=\bar{g}_{i}(0)=\bar{h}_{i}(0)=0, i=1,2, \ldots, n$.

Let $\{r(t), t \geq 0\}$ be a right continuous Markov chain in a complete probability space $(\Omega, \mathscr{F}, \mathscr{P})$ taking values in a finite state space $\mathfrak{M}=\{1,2, \ldots, N\}$ with generator $\Gamma=$ $\left(\gamma_{i j}\right)_{N \times N}$ given by

$$
\begin{aligned}
& P\{r(t+\Delta t)=j \mid r(t)=i\} \\
& \quad= \begin{cases}\gamma_{i j} \Delta t+o(\Delta t), & \text { if } i \neq j, \\
1+\gamma_{i j} \Delta t+o(\Delta t), & \text { if } i=j,\end{cases}
\end{aligned}
$$

where $\Delta t>0$ and $\lim _{\Delta t \rightarrow 0}(o(\Delta t) / \Delta t)=0$. Here, $\gamma_{i j} \geq 0$ is the transition probability rate from $i$ to $j$ if $i \neq j$, while $\gamma_{i i}=$ $-\sum_{j=1, j \neq i}^{N} \gamma_{i j}$.

In this paper, we consider the following neural networks with stochastic noise disturbance, mixed time delays, and Markovian jump parameters, which are actually a modification of the system (6):

$$
\begin{aligned}
& d u(t) \\
& =\left[-C(r(t)) u(t)+W_{0}(r(t)) \bar{f}(u(t))\right. \\
& \quad+W_{1}(r(t)) \bar{g}(u(t-\tau(t))) \\
& \left.\quad+W_{2}(r(t)) \int_{t-\sigma}^{t} \bar{h}(u(s)) d s\right] d t \\
& +\sigma(u(t), u(t-\tau(t)), t, r(t)) d \omega(t),
\end{aligned}
$$

where $u(t), \tau(t), \bar{f}(u(t)), \bar{g}(u(t-\tau(t)))$, and $\bar{h}(u(t))$ have the same meanings as those in (6), $\sigma(u(t), u(t-\tau(t)), t, r(t))$ is noise intensity function vector, and for a fixed system mode $C(r(t)), W_{0}(r(t)), W_{1}(r(t))$, and $W_{2}(r(t))$ are known constant matrices with appropriate dimensions.

For convenience, each possible value of $r(t)$ is denoted by $i ; i \in \mathfrak{M}$ in the sequel. Then we have

$$
\begin{aligned}
C_{i} & =C(r(t)), & W_{0 i}=W_{0}(r(t)), \\
W_{1 i} & =W_{1}(r(t)), & W_{2 i}=W_{2}(r(t)),
\end{aligned}
$$

where $C_{i}, W_{0 i}, W_{1 i}$, and $W_{2 i}$ for any $i \in \mathfrak{M}$ are known constant matrices of appropriate dimensions.

Assume that $\sigma: R^{n} \times R^{n} \times R^{+} \times \mathfrak{M} \rightarrow R^{n}$ is locally Lipschitz continuous and satisfies

$\left(H_{4}\right) \operatorname{trace}\left[\sigma^{T}\left(u_{1}, u_{2}, t, i\right) \sigma\left(u_{1}, u_{2}, t, i\right)\right] \leq u_{1}^{T} R_{1 i} u_{1}+u_{2}^{T} R_{2 i} u_{2}$, for all $u_{1}, u_{2} \in R^{n}$ and $r(t)=i, i \in \mathfrak{M}$, where $R_{1 i}$ and $R_{2 i}$ are known positive definite matrices with appropriate dimensions.

Let $u(t ; \xi)$ denote the state trajectory from the initial data $u(\theta)=\xi(\theta)$ on $-\omega \leq \theta \leq 0$ in $L_{\mathscr{F}_{0}}^{2}\left([-\omega, 0] ; R^{n}\right)$. Clearly, the system (9) admits a trivial solution $u(t ; 0) \equiv 0$ corresponding to the initial data $\xi=0$. For simplicity, we write $u(t ; \xi)=u(t)$.

Before ending this section, we introduce some definitions and lemmas, which will play important roles in the proof of our theorems below.

Definition 5. The equilibrium point of the neural networks (9) is said to be globally exponentially stable in the mean square if, for any $\xi \in L_{\mathscr{F}_{0}^{2}}\left([-\oplus, 0] ; R^{n}\right)$, there exist positive constants $\eta, \mathscr{T}$, and $\pi_{\xi}$ correlated with $\xi$, such that, when $t>\mathscr{T}$, the following inequality holds:

$$
\mathbb{E}\left\{\|u(t ; \xi)\|^{2}\right\} \leq \pi_{\xi} e^{-\eta t}
$$

Definition 6. One has introduces the stochastic LyapunovKrasovskii functional $V \in \mathscr{C}^{2.1}\left(R^{+} \times R^{n} \times \mathfrak{M} ; R^{+}\right)$of the system (9), the weak infinitesimal generator of random process $\mathscr{L} \mathrm{V}$ from $R^{+} \times R^{n} \times \mathfrak{M}$ to $R^{+}$defined by

$$
\begin{gathered}
\mathscr{L} V(t, u(t), i) \\
=\lim _{\Delta t \rightarrow 0^{+}} \frac{1}{\Delta t}[\mathbb{E}\{V(t+\Delta t, u(t+\Delta t), \\
r(t+\Delta t)) \mid u(t), r(t)=i\} \\
-V(t, u(t), r(t)=i)] .
\end{gathered}
$$

Let $\Omega$ be a nonempty, bounded, and open subset of $R^{n}$. The closure of $\Omega$ is denoted by $\bar{\Omega}$, and the boundary of $\Omega$ is denoted by $\partial \Omega$.

Definition 7. Let $f: \bar{\Omega} \rightarrow R^{n}$ be a continuously differentiable function, and $p \in R^{n} \backslash f(\partial \Omega)$; then $\epsilon=\inf _{x \in \partial \Omega}\|f(x)-p\|>0$. Make a continuous function $\Phi:[0,+\infty) \rightarrow R^{1}$, such that

(i) $\exists 0<\sigma<\epsilon^{*} \leq \epsilon / 2$, for all $\gamma \bar{\epsilon}\left(\sigma, \epsilon^{*}\right), \Phi(\gamma)=0$,

(ii) $\int_{R^{n}} \Phi(\|u\|) d u=1$.

Then the topological degree $\operatorname{deg}(f, \Omega, p)$ of $f$ about $p$ on $\Omega$ is defined as

$$
\operatorname{deg}(f, \Omega, p)=\int_{\bar{\Omega}} \Phi(\|f(x)-p\|) J_{f}(x) d x,
$$

where $J_{f}(x)$ is the Jacobi determinant of $f$ at $x$. Let $Z_{f}=\{x \in$ $\left.\Omega \mid J_{f}(x)=0\right\}, p \bar{\epsilon} f(\partial \Omega) \cup f\left(Z_{f}\right)$. Then

$$
\operatorname{deg}(f, \Omega, p)=\sum_{x \in f^{-1}(p)} \operatorname{sgn} J_{f}(x) .
$$

Let $g$ is continuous on $\bar{\Omega}, p \bar{\epsilon} g(\partial \Omega)$. Take a function $f$ which is continuously differentiable on $\bar{\Omega}$, such that

$$
\sup _{x \in \bar{\Omega}}\|g(x)-f(x)\|<\inf _{x \in \partial \Omega}\|g(x)-p\| .
$$

This implies that $p \in R^{n} \backslash f(\partial \Omega)$. Set $\operatorname{deg}(g, \Omega, p)=$ $\operatorname{deg}(f, \Omega, p)$, where $\operatorname{deg}(f, \Omega, p)$ is the topological degree of $f$. Then $\operatorname{deg}(g, \Omega, p)$ is said to be Brouwer degree of the continuous function $g$ about $p$ on $\Omega$. 
Lemma 8 (see [43]). (1) Let $\mathscr{H}:[0,1] \times \bar{\Omega} \rightarrow R^{n}$ be a continuous mapping. For all $\lambda \in[0,1]$, if $p \bar{\epsilon} \mathscr{H}(\lambda, \partial \Omega)$, then Brouwer degree $\operatorname{deg}(\mathscr{H}(\lambda, \cdot), \Omega, p$ ) is constant (for all $\lambda \epsilon$ $[0,1])$. In this case, $\operatorname{deg}(\mathscr{H}(0, \cdot), \Omega, p)=\operatorname{deg}(\mathscr{H}(1, \cdot), \Omega, p)$.

(2) Let $\mathscr{H}: \bar{\Omega} \rightarrow R^{n}$ be a continuous mapping. If $\operatorname{deg}(\mathscr{H}, \Omega, p) \neq 0$, then the equation $\mathscr{H}(x)=p$ has at least $a$ solution in $\Omega$.

Lemma 9 (see [15]). If $f_{i}$ is an $\alpha$-inverse Hölder function, then, for any $\rho_{0} \in R$, one has

$$
\int_{\rho_{0}}^{+\infty}\left[f_{i}(\theta)-f_{i}\left(\rho_{0}\right)\right] d \theta=\int_{\rho_{0}}^{-\infty}\left[f_{i}(\theta)-f_{i}\left(\rho_{0}\right)\right] d \theta=+\infty .
$$

Lemma 10 (see [15]). If $f_{i}$ is an $\alpha$-inverse Hölder function and $f_{i}(0)=0$, then there exist constants $q_{i_{0}}>0$ and $r_{i_{0}}>0$, such that

$$
\left|f_{i}(\theta)\right| \geq q_{i_{0}}|\theta|^{\alpha}, \quad \forall|\theta| \leq r_{i_{0}} .
$$

Moreover,

$$
\left|f_{i}(\theta)\right| \geq q_{i_{0}} r_{i_{0}}^{\alpha}, \quad \forall|\theta| \geq r_{i_{0}} .
$$

Lemma 11 (see [15]). Let $x, y \in R^{n}$, and $G$ is positive definite matrix; then

$$
2 x^{T} y \leq x^{T} G x+y^{T} G^{-1} y .
$$

Lemma 12 (Schur complement [38]). Given constant symmetric matrices $\Upsilon_{1}, \Upsilon_{2}$, and $\Upsilon_{3}$ with appropriate dimensions, where $\Upsilon_{1}^{T}=\Upsilon_{1}$ and $\Upsilon_{2}^{T}=\Upsilon_{2}>0$, then $\Upsilon_{1}+\Upsilon_{3}^{T} \Upsilon_{2}^{-1} \Upsilon_{3}<0$ if and only if

$$
\left(\begin{array}{cc}
\Upsilon_{1} & \Upsilon_{3}^{T} \\
\Upsilon_{3} & -\Upsilon_{2}
\end{array}\right)<0 \quad \text { or }\left(\begin{array}{cc}
\Upsilon_{1} & \Upsilon_{3} \\
\Upsilon_{3}^{T} & -\Upsilon_{2}
\end{array}\right)<0 .
$$

Lemma 13 (Jensen's inequality [38]). For any constant matrix $\Omega \in R^{n \times n}, \Omega=\Omega^{T}>0$, scalar $\gamma>0$, and vector function $\omega:[0, \gamma] \rightarrow R^{n}$, such that the integrations concerned are well defined, then

$$
\frac{1}{\gamma}\left(\int_{0}^{\gamma} \omega(s) d s\right)^{T} \Omega\left(\int_{0}^{\gamma} \omega(s) d s\right) \leq \int_{0}^{\gamma} \omega^{T}(s) \Omega \omega(s) d s .
$$

\section{Main Results}

In this section, stochastic Lyapunov stability theory and a unified LMI approach which is different from the commonly used matrix norm theories (such as the $M$-matrix method) will be developed to establish sufficient conditions for the neural networks system (9) to be globally exponentially stable in the mean square.

Theorem 14. Under assumptions $\left(H_{1}\right)$ and $\left(H_{2}\right)$, the neural network system (9) is globally exponentially stable in the mean square, if, for given $\eta_{i}>0(i \in \mathfrak{M})$, there exist positive definite matrices $S, T$, and $H_{i}(i \in \mathfrak{M})$, positive definite diagonal matrix $P$, and positive scalars $\lambda_{i},(i \in \mathfrak{M})$ such that the following LMIs are satisfied:

$$
\begin{aligned}
& H_{i}<\lambda_{i} I, \\
& \Pi_{i}=\left(\begin{array}{cccc}
\Xi_{1 i} & H_{i} W_{0 i}+\eta_{i} P-P C_{i} & H_{i} W_{1 i} & H_{i} W_{2 i} \\
* & \Xi_{2 i} & P W_{1 i} & P W_{2 i} \\
* & * & -e^{-\eta_{i} \tau} S & 0 \\
* & * & * & -\frac{1}{\sigma} T
\end{array}\right)<0,
\end{aligned}
$$

where

$$
\begin{aligned}
& \Xi_{1 i}=-2 H_{i} C_{i}+\lambda_{i} R_{1 i}+\eta_{i} H_{i}+\frac{\lambda_{i}}{1-\tau} e^{\eta_{i} \bar{\tau}} R_{2 i}+\sum_{j=1}^{N} \gamma_{i j} H_{j}, \\
& \Xi_{2 i}=P W_{0 i}+W_{0 i}^{T} P+\sigma K^{2} T+\frac{1}{1-\tau} E^{2} S .
\end{aligned}
$$

Remark 15. From condition (23), it easily follows that condition (5) holds. Hence, under the assumptions of Theorem 14, the system (9) admits an unique equilibrium point $u^{*}=$ $(0,0, \ldots, 0)^{T}$.

Proof. Define a positive definite Lyapunov-Krasovskii functional $V(t, u(t), i) \in \mathscr{C}^{2.1}\left(R^{+} \times R^{n} \times \mathfrak{M} ; R^{+}\right)$as follows:

$$
V(t, u(t), i)=\sum_{j=1}^{5} V_{j}(t, u(t), i),
$$

where

$$
\begin{aligned}
& V_{1}(t, u(t), i)=e^{\eta_{i} t} u^{T}(t) H_{i} u(t), \\
& V_{2}(t, u(t), i)=\frac{\lambda_{i}}{1-\tau} \int_{t-\tau(t)}^{t} e^{\eta_{i}(s+\tau(s))} u^{T}(s) R_{2 i} u(s) d s, \\
& V_{3}(t, u(t), i)=\frac{1}{1-\tau} \int_{t-\tau(t)}^{t} e^{\eta_{i} s} \bar{g}^{T}(u(s)) S \bar{g}(u(s)) d s, \\
& V_{4}(t, u(t), i)=\int_{-\sigma}^{0} \int_{t+s}^{t} e^{\eta_{i} \theta} \bar{h}^{T}(u(\theta)) T \bar{h}(u(\theta)) d \theta d s, \\
& V_{5}(t, u(t), i)=2 e^{\eta_{i} t} \sum_{j=1}^{n} p_{j} \int_{0}^{u_{j}(t)} \bar{f}_{j}(\theta) d \theta .
\end{aligned}
$$

By assumption $\left(H_{4}\right)$ and condition (22), we obtain

$$
\begin{aligned}
\operatorname{trace}\left(\sigma^{T}(t, u(t), u(t-\tau(t)), i)\right. \\
\left.\times H_{i} \sigma(t, u(t), u(t-\tau(t))), i\right) \\
\leq \lambda_{i}\left[u^{T}(t) R_{1 i} u(t)+u^{T}(t-\tau(t)) R_{2 i} u(t-\tau(t))\right] .
\end{aligned}
$$


Hence, using Lemmas 11 and 13, it follows from (9) and Definition 6 that

$$
\begin{aligned}
& \mathscr{L} V_{1}=\eta_{i} e^{\eta_{i} t} u^{T}(t) H_{i} u(t)+2 e^{\eta_{i} t} u^{T}(t) H_{i} \\
& \times\left[-C_{i} u(t)+W_{0 i} \bar{f}(u(t))+W_{1 i} \bar{g}(u(t-\tau(t)))\right. \\
& \left.+W_{2 i} \int_{t-\sigma}^{t} \bar{h}(u(s)) d s\right]+e^{\eta_{i} t} u^{T}(t) \sum_{j=1}^{N} \gamma_{i j} H_{j} u(t) \\
& +e^{\eta_{i} t} \operatorname{trace}\left(\sigma^{T}(t, u(t), u(t-\tau(t)), i)\right. \\
& \left.\times H_{i} \sigma(t, u(t), u(t-\tau(t))), i\right) \\
& \leq e^{\eta_{i} t} u^{T}(t)\left(-H_{i} C_{i}-C_{i} H_{i}+\lambda_{i} R_{1 i}+\eta_{i} H_{i}\right. \\
& \left.+\sum_{j=1}^{N} \gamma_{i j} H_{j}\right) u(t) \\
& +2 e^{\eta_{i} t} u^{T}(t) H_{i} W_{0 i} \bar{f}(u(t)) \\
& +2 e^{\eta_{i} t} u^{T}(t) H_{i} W_{1 i} \bar{g}(u(t-\tau(t))) \\
& +2 e^{\eta_{i} t} u^{T}(t) H_{i} W_{2 i} \int_{t-\sigma}^{t} \bar{h}(u(s)) d s \\
& +\lambda_{i} e^{\eta_{i} t} u^{T}(t-\tau(t)) R_{2 i} u(t-\tau(t)),
\end{aligned}
$$$$
\mathscr{L} V_{2} \leq \frac{\lambda_{i}}{1-\tau} e^{\eta_{i}(t+\tau(t))} u^{T}(t) R_{2 i} u(t)
$$$$
-\frac{\lambda_{i}}{1-\tau}(1-\dot{\tau}(t)) e^{\eta_{i} t} u^{T}(t-\tau(t)) R_{2 i} u(t-\tau(t))
$$$$
\leq \frac{\lambda_{i}}{1-\tau} e^{\eta_{i}(t+\bar{\tau})} u^{T}(t) R_{2 i} u(t)
$$$$
-\lambda_{i} e^{\eta_{i} t} u^{T}(t-\tau(t)) R_{2 i} u(t-\tau(t)),
$$

$$
\begin{aligned}
\mathscr{L} V_{3} \leq & \frac{1}{1-\tau} e^{\eta_{i} t} \bar{g}^{T}(u(t)) S \bar{g}(u(t)) \\
& -e^{\eta_{i}(t-\tau(t))} \bar{g}^{T}(u(t-\tau(t))) S \bar{g}(u(t-\tau(t))) \\
\leq & \frac{1}{1-\tau} e^{\eta_{i} t} \bar{f}^{T}(u(t)) E^{2} S \bar{f}(u(t)) \\
& -e^{\eta_{i}(t-\bar{\tau})} \bar{g}^{T}(u(t-\tau(t))) S \bar{g}(u(t-\tau(t))), \\
\mathscr{L} V_{4} \leq & \sigma e^{\eta_{i} t} \bar{h}^{T}(u(t)) T \bar{h}(u(t)) \\
& -\int_{t-\sigma}^{t} e^{\eta_{i} s} \bar{h}^{T}(u(s)) T \bar{h}(u(s)) d s
\end{aligned}
$$

$$
\begin{aligned}
\mathscr{L} V_{5}= & 2 \eta_{i} e^{\eta_{i} t} \sum_{j=1}^{n} p_{j} \int_{0}^{u_{j}(t)} \bar{f}_{j}(\theta) d \theta \\
+ & +2 e^{\eta_{i} t} \bar{f}^{T}(u(t)) P\left[-C_{i} u(t)+W_{0 i} \bar{f}(u(t))\right. \\
& +W_{1 i} \bar{g}(u(t-\tau(t))) \\
& \left.+W_{2 i} \int_{t-\sigma}^{t} \bar{h}(u(s)) d s\right] \\
\leq & 2 e^{\eta_{i} t} \bar{f}^{T}(u(t))\left(\eta_{i} P-P C_{i}\right) u(t) \\
+ & 2 e^{\eta_{i} t} \bar{f}^{T}(u(t)) P W_{0 i} \bar{f}(u(t)) \\
+ & 2 e^{\eta_{i} t} \bar{f}^{T}(u(t)) P W_{1 i} \bar{g}(u(t-\tau(t))) \\
+ & 2 e^{\eta_{i} t} \bar{f}^{T}(u(t)) P W_{2 i} \int_{t-\sigma}^{t} \bar{h}(u(s)) d s .
\end{aligned}
$$

By combing (28)-(32), we can obtain that

$$
\mathscr{L} V(t, u(t), i) \leq e^{\eta_{i} t} \psi^{T}(t) \Pi_{i} \psi(t),
$$

where $\psi^{T}(t)=\left[u^{T}(t), \bar{f}^{T}(u(t)), \bar{g}^{T}(u(t-\tau(t))),\left(\int_{t-\sigma}^{t} \bar{h}(u(s))\right.\right.$ $\left.d s)^{T}\right]$.

Let $\delta=\min _{i \in S} \lambda_{\min }\left(-\Pi_{i}\right)$. From condition (23), it is easy to see that $\delta>0$. This fact, together with (33), yields

$$
\mathscr{L} V(t, u(t), i) \leq-\delta e^{\eta_{i} t}\|u(t)\|^{2} .
$$

Hence, it follows from the definition of $V(t, u(t), i)$, generalized Itôs's formula, and (34) that

$$
\begin{aligned}
& \lambda_{\min }\left(H_{i}\right) \mathbb{E}\left\{\|u(t)\|^{2}\right\} \\
& \leq \mathbb{E}\left[u^{T}(t) H_{i} u(t)\right] \\
& \leq e^{-\eta_{i} t} \mathbb{E} V(t, u(t), i) \\
& =e^{-\eta_{i} t}\{\mathbb{E} V(0, u(0), r(0)) \\
& \left.+\mathbb{E}\left[\int_{0}^{t} \mathscr{L} V(s, u(s), r(s))\right]\right\} \\
& \leq e^{-\eta_{i} t}\{\mathbb{E} V(0, u(0), r(0)) \\
& \left.+\mathbb{E}\left[\int_{0}^{t}-\delta e^{\eta_{i} s}\|u(s)\|^{2} d s\right]\right\} \\
& \leq e^{-\eta_{i} t} \mathbb{E} V(0, u(0), r(0)) .
\end{aligned}
$$


By (35), we can get that $\lim _{t \rightarrow+\infty} \mathbb{E}\left\{\|u(t)\|^{2}\right\}=0$. Furthermore,

$$
\lim _{t \rightarrow+\infty} \mathbb{E}\left\{\left|u_{j}(t)\right|^{2}\right\}=0, \quad j=1,2, \ldots, n .
$$

For $\bar{f}_{j}(\theta)$, by Lemma 10 , there exist constants $q_{j_{0}}>0$ and $r_{j_{0}}>0$ such that

$$
\left|\bar{f}_{j}(\theta)\right| \geq q_{j_{0}}|\theta|^{\alpha}, \quad \forall|\theta| \leq r_{j_{0}}, j=1,2, \ldots, n .
$$

By (36), there exists a scalar $\mathscr{T}>0$, when $t \geq \mathscr{T}, \mathbb{E}\left\{u_{j}(t)\right\} \in$ $\left[-\bar{r}_{0}, \bar{r}_{0}\right], j=1,2, \ldots, n$, where $\bar{r}_{0}=\min _{1 \leq j \leq n} r_{j_{0}}$. Hence, when $t \geq \mathscr{T}$, we have

$$
\begin{aligned}
e^{-\eta_{i} t} \mathbb{E} V(0, u(0), r(0)) & \geq \mathbb{E}\left[2 \sum_{j=1}^{n} p_{j} \int_{0}^{u_{j}(t)} \bar{f}_{j}(\theta) d \theta\right] \\
& \geq 2 \mathbb{E}\left[\sum_{j=1}^{n} p_{j} \int_{0}^{\left|u_{j}(t)\right|} q_{j_{0}}|\theta|^{\alpha} d \theta\right] \\
& \geq \frac{2 p q_{0}}{\alpha+1}\left\{\max _{1 \leq j \leq n} \mathbb{E}\left\{\left\|u_{j}(t)\right\|^{2}\right\}\right\}^{(\alpha+1) / 2},
\end{aligned}
$$

where $p=\min _{1 \leq j \leq n} p_{i}, q_{0}=\min _{1 \leq j \leq n} q_{j_{0}}$. By (38), we get

$$
\left\{\max _{1 \leq j \leq n} \mathbb{E}\left\{\left\|u_{j}(t)\right\|^{2}\right\}\right\}^{(\alpha+1) / 2} \leq \frac{\alpha+1}{2 p q_{0}} \mathbb{E} V(0, u(0), r(0)) e^{-\eta_{i} t} .
$$

Hence,

$$
\mathbb{E}\left\{\|u\|^{2}\right\} \leq n\left[\frac{\alpha+1}{2 p q_{0}} \mathbb{E} V(0, u(0), r(0))\right]^{2 /(\alpha+1)} e^{\left(-2 \eta_{i} /(\alpha+1)\right) t},
$$

where

$$
\begin{aligned}
\mathbb{E} V(0, u(0), & r(0)) \\
=\mathbb{E}\left\{u^{T}(0) H_{i} u(0)+\frac{\lambda_{i}}{1-\tau}\right. & \\
\quad & \int_{-\tau(0)}^{0} e^{\eta_{i}(s+\tau(s))} u^{T}(s) R_{2 i} u(s) d s \\
& +\frac{1}{1-\tau} \int_{-\tau(0)}^{0} e^{\eta_{i} s} \bar{g}^{T}(u(s)) S \bar{g}(u(s)) d s \\
& +\int_{-\sigma}^{0} \int_{s}^{0} e^{\eta_{i} \theta \bar{h}^{T}}(u(\theta)) T \bar{h}(u(\theta)) d \theta d s \\
& \left.+2 \sum_{i=1}^{n} p_{j} \int_{0}^{u_{j}(0)} \bar{f}_{j}(\theta) d \theta\right\} \leq \lambda_{\max }\left(H_{i}\right) \mathbb{E}\|\xi\|^{2}
\end{aligned}
$$

$$
\begin{aligned}
& +\lambda_{\max }\left(R_{2 i}\right) \frac{\lambda_{i}}{1-\tau} \frac{e^{\eta_{i} \bar{\tau}}-1}{\eta_{i}(1+\tau)} \mathbb{E}\|\xi\|^{2} \\
& +\lambda_{\max }(S) \frac{1}{1-\tau} \frac{1-e^{-\eta_{i} \bar{\tau}}}{\eta_{i}} \mathbb{E}\|\bar{g}(\xi)\|^{2} \\
& +\lambda_{\max }(T) \sigma \frac{1-e^{-\eta_{i} \bar{\tau}}}{\eta_{i}} \mathbb{E}\|\bar{h}(\xi)\|^{2}+2 P \mathbb{E}|\xi \bar{f}(\xi)| .
\end{aligned}
$$

Let

$$
\begin{aligned}
\pi_{\xi i}=n\left\{\frac{\alpha+1}{2 p q_{0}}(\right. & \lambda_{\max }\left(H_{i}\right) \mathbb{E}\|\xi\|^{2} \\
& +\lambda_{\max }\left(R_{2 i}\right) \frac{\lambda_{i}}{1-\tau} \frac{e^{\eta_{i} \bar{\tau}}-1}{\eta_{i}(1+\tau)} \mathbb{E}\|\xi\|^{2} \\
& +\lambda_{\max }(S) \frac{1}{1-\tau} \frac{1-e^{-\eta_{i} \bar{\tau}}}{\eta_{i}} \mathbb{E}\|\bar{g}(\xi)\|^{2} \\
& +\lambda_{\max }(T) \sigma \frac{1-e^{-\eta_{i} \bar{\tau}}}{\eta_{i}} \mathbb{E}\|\bar{h}(\xi)\|^{2} \\
& +2 P \mathbb{E}|\xi \bar{f}(\xi)|)\}^{2 /(\alpha+1)}, \\
\pi_{\xi}= & \max _{i \in \mathfrak{M}} \pi_{\xi i}, \quad \eta=\min _{i \in \mathfrak{M}} \eta_{i} .
\end{aligned}
$$

It follows from (41) and (42) that

$$
\mathbb{E}\left\{\|u(t)\|^{2}\right\} \leq \pi_{\xi} e^{(-2 \eta /(\alpha+1)) t} .
$$

Therefore, by Definition 5 and (43), we see that the equilibrium point of the neural networks (9) is globally exponentially stable in the mean square. This completes the proof.

Remark 16. To the best of our knowledge, the global exponential stability criteria applying $\alpha$-inverse Hölder activation functions for stochastic neural networks with Markovian jump parameters and mixed time delays have not been discussed in the existing literature. This paper reports new idea and some sufficient exponential stability conditions of neural networks with stochastic noise disturbance, mixed time delays, $\alpha$-inverse Hölder functions, and Markovian jump parameters, which generalize and improve the outcomes in [14-19].

Remark 17. The criterion given in Theorem 14 is dependent on the time delay. It is well known that the delay-dependent criteria are less conservative than delay-independent criteria, particularly when the delay is small.

Based on Theorem 14, the following results can be obtained easily. 
Case 1. If we do not take the Markovian jumping into account, then the neural network system (9) is simplified to

$$
\begin{aligned}
d u(t)= & {\left[-C u(t)+W_{0} \bar{f}(u(t))+W_{1} \bar{g}(u(t-\tau(t)))\right.} \\
& \left.+W_{2} \int_{t-\sigma}^{t} \bar{h}(u(s)) d s\right] d t \\
& +\sigma(u(t), u(t-\tau(t), t)) d \omega(t) .
\end{aligned}
$$

Corollary 18. Under assumptions $\left(H_{1}\right)$ and $\left(H_{2}\right)$, the neural network system (44) is globally exponentially stable in the mean square, if, for given $\eta>0$, there exist positive definite matrices $S, T$, and $H$, positive definite diagonal matrix $P$, and positive scalar $\lambda$, such that the following LMIs are satisfied:

$$
\Pi=\left(\begin{array}{cccc}
\Xi_{1} & H W_{0}+\eta P-P C & H W_{1} & H W_{2} \\
* & \Xi_{2} & P W_{1} & P W_{2} \\
* & * & -e^{-\eta \bar{\tau}} S & 0 \\
* & * & * & -\frac{1}{\sigma} T
\end{array}\right)<0,
$$

where

$$
\begin{aligned}
& \Xi_{1}=-2 H C+\lambda_{i} R_{1}+\eta_{i} H+\frac{\lambda_{i}}{1-\tau} e^{\eta_{i} \bar{\tau}} R_{2}, \\
& \Xi_{2}=P W_{0}+W_{0}^{T} P+\sigma K^{2} T+\frac{1}{1-\tau} E^{2} S .
\end{aligned}
$$

Case 2. If there are no stochastic disturbances in the system (9), then the neural networks are simplified to

$$
\begin{aligned}
\dot{u}(t)= & -C(r(t)) u(t)+W_{0}(r(t)) \bar{f}(u(t)) \\
& +W_{1}(r(t)) \bar{g}(u(t-\tau(t))) \\
& +W_{2}(r(t)) \int_{t-\sigma}^{t} \bar{h}(u(s)) d s .
\end{aligned}
$$

Corollary 19. Under assumptions $\left(H_{1}\right)$ and $\left(H_{2}\right)$, the neural network system (47) is globally exponentially stable in the mean square, if, for given $\eta_{i}>0(i \in \mathfrak{M})$, there exist positive definite matrices $S, T$, and $H_{i}(i \in \mathfrak{M})$, positive definite diagonal matrix $P$, and positive scalars $\lambda_{i}(i \in \mathfrak{M})$, such that the following LMIs are satisfied:

$$
\begin{gathered}
H_{i}<\lambda_{i} I, \\
\Pi_{i}=\left(\begin{array}{cccc}
\Xi_{1 i} & H_{i} W_{0 i}+\eta_{i} P-P C_{i} & H_{i} W_{1 i} & H_{i} W_{2 i} \\
* & \Xi_{2 i} & P W_{1 i} & P W_{2 i} \\
* & * & -e^{-\eta_{i} \tau} S & 0 \\
* & * & * & -\frac{1}{\sigma} T
\end{array}\right)
\end{gathered}
$$$$
<0
$$

where

$$
\begin{aligned}
& \Xi_{1 i}=-2 H_{i} C_{i}+\eta_{i} H_{i}+\sum_{j=1}^{N} \gamma_{i j} H_{j}, \\
& \Xi_{2 i}=P W_{0 i}+W_{0 i}^{T} P+\sigma K^{2} T+\frac{1}{1-\tau} E^{2} S .
\end{aligned}
$$

\section{Illustrative Examples}

In this section, we provide two numerical examples to demonstrate the effectiveness of the theoretical results above.

Example 1. Consider the second-order stochastic neural network (9) with $u(t)=\left(u_{1}(t), u_{2}(t)\right)^{T} ; \omega(t)$ is a second-order Brownian motion, and $r(t)$ is a right-continuous Markov chain taking values in $\mathfrak{M}=\{1,2\}$ with generator

$$
\Gamma=\left(\begin{array}{cc}
-2 & 2 \\
4 & -4
\end{array}\right)
$$

For the two operating conditions (modes), the associated data are

$$
\begin{array}{cl}
C_{1}=\left(\begin{array}{ll}
1 & 0 \\
0 & 1
\end{array}\right), & W_{01}=\left(\begin{array}{cc}
-10 & 1 \\
-2 & 6
\end{array}\right), \\
W_{11}=\left(\begin{array}{cc}
-1 & 2 \\
1 & 1
\end{array}\right), & W_{21}=\left(\begin{array}{cc}
-1 & 1 \\
-3 & -1
\end{array}\right), \\
C_{2}=\left(\begin{array}{ll}
2 & 0 \\
0 & 2
\end{array}\right), & W_{02}=\left(\begin{array}{cc}
-10 & -2 \\
2 & -6
\end{array}\right), \\
W_{12}=\left(\begin{array}{cc}
-2 & 2 \\
1 & -3
\end{array}\right), & W_{22}=\left(\begin{array}{ll}
2 & -1 \\
2 & -2
\end{array}\right) .
\end{array}
$$

Taking

$$
\begin{aligned}
& \sigma(t, u(t), u(t-\tau(t)), 1) \\
& \quad=\left(\begin{array}{cc}
0.2 u_{1}(t)+0.1 u_{1}(t-\tau(t)) & 0 \\
0 & 0.2 u_{2}(t)+0.1 u_{2}(t-\tau(t))
\end{array}\right), \\
& \sigma(t, u(t), u(t-\tau(t)), 2) \\
& \quad=\left(\begin{array}{cc}
0.3 u_{1}(t)+0.4 u_{1}(t-\tau(t)) & 0.3 u_{2}(t)+0.4 u_{2}(t-\tau(t))
\end{array}\right),
\end{aligned}
$$

assumption $\left(H_{4}\right)$ is satisfied with $R_{11}=R_{12}=0.08 I_{2}, R_{21}=$ $R_{22}=0.02 I_{2}$. Set $f_{i}(x)=\sinh (x), g_{i}(x)=x$, and $h_{i}(x)=$ $\sin x, i=1,2$. It is easy to see that, for any $\theta, s \in R$ with $\theta<s$, there exists a scalar $\nu \in(\theta, s)$ such that

$$
\frac{f_{i}(\theta)-f_{i}(s)}{\theta-s}=\frac{\sinh (\theta)-\sinh (s)}{\theta-s}=\cosh (\nu) \geq 1 .
$$

So $f_{i}(x), i=1,2$, are 1 -inverse Hölder function. In addition, for any $\theta, s \in R$, it is easy to verify that

$$
\begin{aligned}
\left|h_{i}(\theta)-h_{i}(s)\right| & =|\sin (\theta)-\sin (s)| \leq\left|g_{i}(\theta)-g_{i}(s)\right|=|\theta-s| \\
& \leq\left|f_{i}(\theta)-f_{i}(s)\right|=|\sinh (\theta)-\sinh (s)| .
\end{aligned}
$$

That is, the activation functions $f_{i}(x), g_{i}(x)$, and $h_{i}(x)(i=$ $1,2)$ satisfy assumption $\left(H_{2}\right)$ with $E=K=I$. For the model with $\tau(t)=0.1-0.1 \cos t, \sigma=0.5$. We choose $\eta_{1}=0.5, \eta_{2}=$ 0.4. Using Theorem 14 and MATLAB LMI control toolbox, we can find that the neural network (9) is globally exponentially 
stable in the mean square and the feasible solutions of the LMIs (22) and (23) are given as follows:

$$
\begin{array}{cc}
P=\left(\begin{array}{cc}
1.3084 & 0 \\
0 & 3.3380
\end{array}\right), \quad S=\left(\begin{array}{cc}
3.5409 & -1.5268 \\
-1.5268 & 6.6849
\end{array}\right), \\
T=\left(\begin{array}{cc}
20.3336 & 7.0607 \\
7.0607 & 4.2001
\end{array}\right), \quad H_{1}=\left(\begin{array}{cc}
0.4597 & 0.1099 \\
0.1099 & 0.5309
\end{array}\right), \\
H_{2}=\left(\begin{array}{ll}
0.4275 & 0.2318 \\
0.2318 & 0.5248
\end{array}\right), \\
\lambda_{1}=1.0067, \quad \lambda_{2}=0.9967 .
\end{array}
$$

Figure 1 shows Markovian chain generated by probability transition matrix corresponding to the generator (50) with $\Delta t=0.01, \gamma(0)=2$. Figure 2 shows the state response of the neural network with the initial condition $\varphi(t)=$ $(-0.2,0.2)^{T}, t \in[-0.2,0]$ in this example. The simulation results imply that neural network in this example is globally exponentially stable in the mean square.

Example 2. Consider the three-order stochastic neural network (9) with $u(t)=\left(u_{1}(t), u_{2}(t), u_{3}(t)\right)^{T} ; \omega(t)$ is a threeorder Brownian motion, and $r(t)$ is a right-continuous Markov chain taking values in $\mathfrak{M}=\{1,2,3\}$ with generator

$$
\Gamma=\left(\begin{array}{ccc}
1 & 1 & -2 \\
2 & -4 & 2 \\
-1 & 2 & -1
\end{array}\right)
$$

For the three operating conditions (modes), the associated data are

$$
\begin{gathered}
C_{1}=\left(\begin{array}{lll}
3 & 0 & 0 \\
0 & 3 & 0 \\
0 & 0 & 3
\end{array}\right), \quad C_{2}=\left(\begin{array}{lll}
2 & 0 & 0 \\
0 & 2 & 0 \\
0 & 0 & 2
\end{array}\right), \\
C_{3}=\left(\begin{array}{lll}
1 & 0 & 0 \\
0 & 1 & 0 \\
0 & 0 & 1
\end{array}\right), \quad W_{01}=\left(\begin{array}{ccc}
-12 & 1 & 2 \\
2 & -12 & -1 \\
1 & 2 & -32
\end{array}\right), \\
W_{11}=\left(\begin{array}{ccc}
1 & 2 & 1 \\
1 & 0 & 1 \\
-1 & 2 & 0
\end{array}\right), \quad W_{21}=\left(\begin{array}{ccc}
1 & -1 & 0 \\
1 & 0 & -1 \\
0 & -1 & 1
\end{array}\right), \\
W_{02}=\left(\begin{array}{ccc}
-31 & -1 & 2 \\
1 & -25 & 3 \\
1 & 2 & -33
\end{array}\right), \quad W_{12}=\left(\begin{array}{ccc}
1 & 2 & 8 \\
1 & 1 & 0 \\
1 & 2 & 1
\end{array}\right), \\
W_{22}=\left(\begin{array}{ccc}
1 & 2 & 1 \\
1 & 0 & 2 \\
1 & 1 & 2
\end{array}\right), \quad W_{03}=\left(\begin{array}{ccc}
-12 & -2 & -2 \\
1 & -41 & -3 \\
1 & 2 & -43
\end{array}\right), \\
W_{13}=\left(\begin{array}{ccc}
1 & 0 & 4 \\
-1 & 1 & 2 \\
-6 & 2 & 1
\end{array}\right), \quad W_{23}=\left(\begin{array}{ccc}
1 & 2 & -5 \\
1 & 1 & 0 \\
1 & -3 & 1
\end{array}\right) .
\end{gathered}
$$

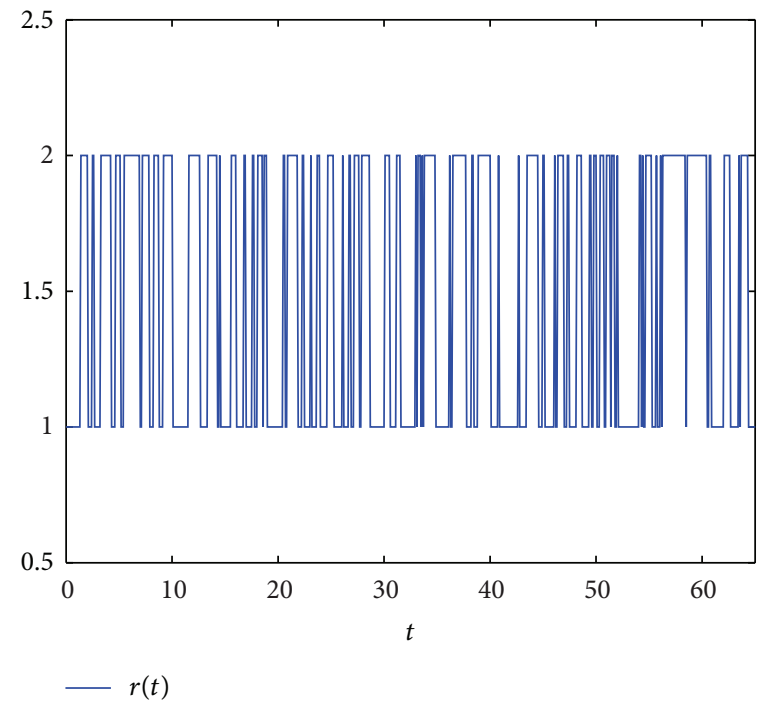

FIGURE 1: Markovian chain generated by probability transition matrix corresponding to the generator (50) with $\Delta t=0.01, \gamma(0)=2$.

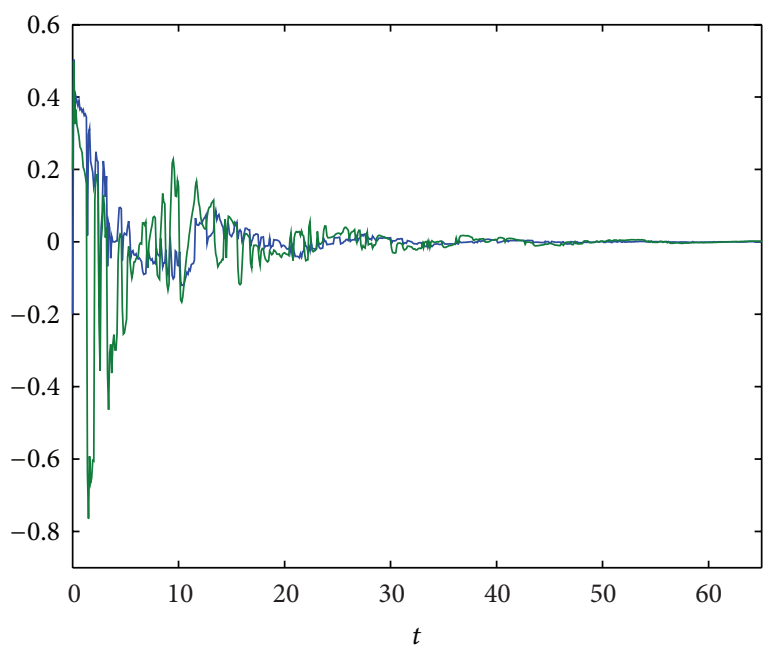

FIGURE 2: State response of the neural network under the Markov chain depicted in Figure 1 in Example 1.

Taking

$$
\begin{aligned}
& \sigma(t, u(t), u(t-\tau(t)), 1) \\
& \quad=\left(\begin{array}{cc}
0.2 u_{1}(t)+0.1 u_{1}(t-\tau(t)) & 0 \\
0 & 0.2 u_{2}(t)+0.1 u_{2}(t-\tau(t))
\end{array}\right), \\
& \sigma(t, u(t), u(t-\tau(t)), 2) \\
& \quad=\left(\begin{array}{cc}
0.3 u_{1}(t)+0.4 u_{1}(t-\tau(t)) & 0.3 u_{2}(t)+0.4 u_{2}(t-\tau(t))
\end{array}\right),
\end{aligned}
$$




$$
\begin{aligned}
\sigma & (t, u(t), u(t-\tau(t)), 3) \\
& =\left(\begin{array}{cc}
0.2 u_{1}(t)+0.5 u_{1}(t-\tau(t)) & 0 \\
0 & 0.5 u_{2}(t)+0.5 u_{2}(t-\tau(t))
\end{array}\right),
\end{aligned}
$$

assumption $\left(H_{4}\right)$ is satisfied with $R_{11}=R_{12}=R_{13}=0.08 I_{2}$, $R_{21}=R_{22}=R_{23}=0.02 I_{2}$.

Set $f_{i}(x)=x^{3}+x ; g_{i}(x)$ and $h_{i}(x), i=1,2,3$, are the functions in Example 1. That is, the activation functions $f_{i}(x)$, $g_{i}(x)$, and $h_{i}(x)(i=1,2,3)$ satisfy assumption $\left(H_{2}\right)$ with $E=K=I$. For the model with $\tau(t)=0.15-0.15$ cos $t$, $\sigma=0.5$. We choose $\eta_{1}=0.2, \eta_{2}=0.1$, and $\eta_{2}=0.3$. Using Theorem 14 and MATLAB LMI control toolbox, we can find that the neural network (9) is globally exponentially stable in the mean square and the feasible solutions of the LMIs (22) and (23) are given as follows:

$$
\begin{aligned}
& P=\left(\begin{array}{ccc}
0.0175 & 0 & 0 \\
0 & 0.0899 & 0 \\
0 & 0 & 0.0915
\end{array}\right), \\
& S=\left(\begin{array}{ccc}
0.5204 & -0.0719 & 0.0047 \\
-0.0719 & 0.5359 & -0.0107 \\
0.0047 & -0.0107 & 1.5105
\end{array}\right) \\
& T=\left(\begin{array}{ccc}
0.5972 & -0.0106 & -0.0601 \\
-0.0106 & 0.6667 & -0.1291 \\
-0.0601 & -0.1291 & 0.9822
\end{array}\right) \text {, } \\
& H_{1}=\left(\begin{array}{ccc}
0.1763 & -0.0082 & 0.0027 \\
-0.0082 & 0.2530 & -0.0018 \\
0.0027 & -0.0018 & 0.1248
\end{array}\right) \text {, } \\
& H_{2}=\left(\begin{array}{ccc}
0.1007 & -0.0078 & 0.0017 \\
-0.0078 & 0.1589 & 0.0034 \\
0.0017 & 0.0034 & 0.1070
\end{array}\right) \text {, } \\
& H_{3}=\left(\begin{array}{ccc}
0.1678 & -0.0123 & 0.0051 \\
-0.0123 & 0.1237 & -0.0039 \\
0.0051 & -0.0039 & 0.0897
\end{array}\right) \text {, } \\
& \lambda_{1}=1.0067, \quad \lambda_{2}=0.7967, \quad \lambda_{3}=0.8871 .
\end{aligned}
$$

Figure 3 shows Markovian chain generated by probability transition matrix corresponding to the generator (50) with $\Delta t=0.01, \gamma(0)=2$. Figure 4 shows the state response of the neural network with the initial condition $\varphi(t)=$ $(0.2,-0.2,-0.2)^{T}, t \in[-0.3,0]$ in this example. The simulation results imply that neural network in this example is globally exponentially stable in the mean square.

\section{Conclusion}

In this paper, we have dealt with the global exponential stability issue for a class of stochastic neural networks with $\alpha$ inverse Hölder activation functions, Markovian jump parameters, and mixed time delays. The delay-dependent sufficient conditions have been achieved in terms of LMIs to ensure the considered neural network with noise perturbations to be

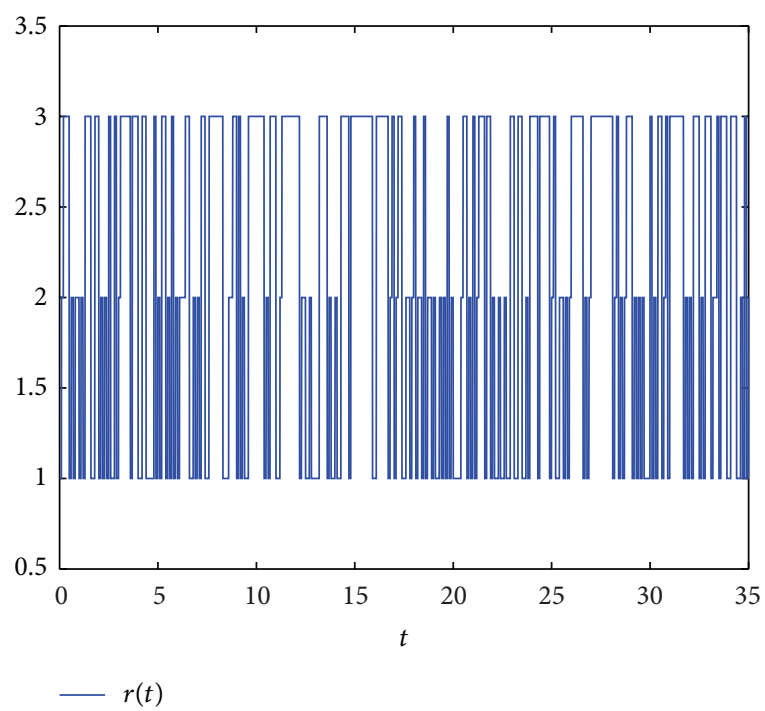

FIGURE 3: Markovian chain generated by probability transition matrix corresponding to the generator (50) with $\Delta t=0.01, \gamma(0)=2$.

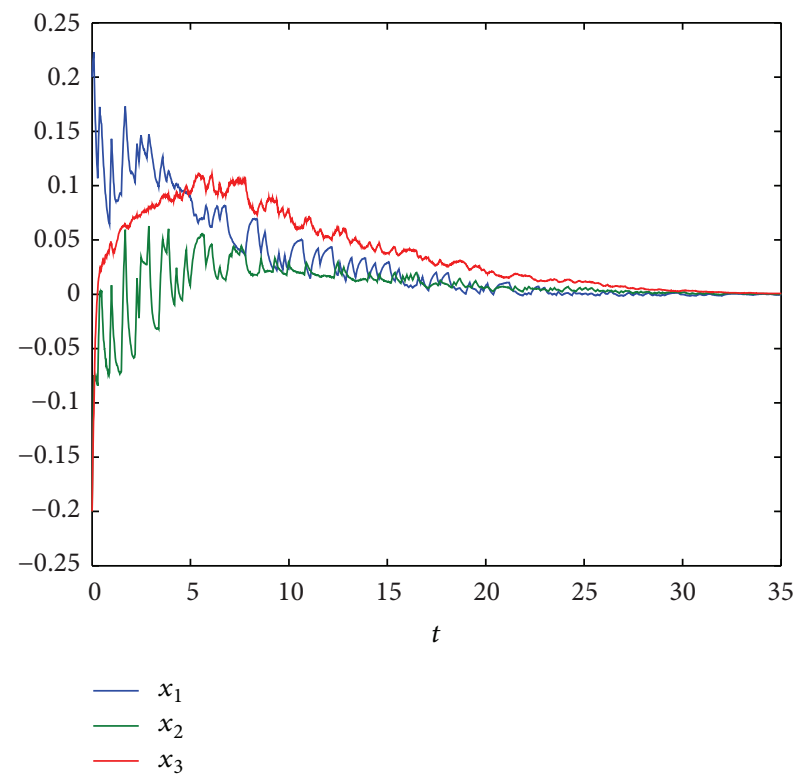

FIGURE 4: State response of the neural network under the Markov chain depicted in Figure 3 in Example 1.

globally exponentially stable in the mean square. The criteria obtained can be tested easily by using the MATLAB LMI toolbox and applied in practice engineering. Two numerical simulation examples have been given to check the usefulness of the results presented in this paper.

When neuron activation functions are non-Lipschitz functions, it is possible that the neural network system has no global solution and equilibrium point. This leads to difficulty in solving the stability problem for various stochastic neural networks with non-Lipschitz activation functions. In the future, the stability problem for the Markovian jumping 
stochastic neural networks with other non-Lipschitz activation functions will be expected to be solved.

\section{Appendix}

\section{The Proof of Theorem 4}

Proof. Let $\mathscr{H}(x)=C x-W_{0} f(x)-W_{1} g(x)-\sigma W_{2} h(x)-J$. $x^{*} \in R^{n}$ is an equilibrium point of the system (4) if and only if $\mathscr{H}\left(x^{*}\right)=0$. Rewrite $\mathscr{H}(x)$ as

$$
\mathscr{H}(x)=C x-W_{0} \widehat{f}(x)-W_{1} \widehat{g}(x)-\sigma W_{2} \widehat{h}(x)+\mathscr{H}(0),
$$

where $\widehat{f}(x)=f(x)-f(0), \widehat{g}(x)=g(x)-g(0), \widehat{h}(x)=h(x)-$ $h(0)$, and $\widehat{f}(0)=\widehat{g}(0)=\widehat{h}(0)=0$. By assumption $\left(H_{2}\right)$, we can see that the following inequalities hold: $\left|\widehat{g}_{i}(s)\right| \leq e_{i}\left|\widehat{f}_{i}(s)\right|$, $\left|\widehat{h}_{i}(s)\right| \leq k_{i}\left|\widehat{f}_{i}(s)\right|$. According to assumption $\left(H_{1}\right), \widehat{f}_{i}$ is also an $\alpha$-inverse Hölder function, and $x_{i} \widehat{f}_{i}\left(x_{i}\right)>0 \quad\left(x_{i} \neq 0\right)$. For the scalar $\gamma>0$, set $\Omega_{\gamma}=\left\{x \in R^{n}:\|x\|<\gamma\right\}$. Define the mapping $\mathscr{H}:[0,1] \times \bar{\Omega}_{\gamma} \rightarrow R^{n}$ as

$$
\begin{aligned}
\mathscr{H}(\lambda, x)= & C x-\lambda\left[W_{0} \widehat{f}(x)+W_{1} \hat{g}(x)+\sigma W_{2} \widehat{h}(x)\right] \\
& +\lambda \mathscr{H}(0),
\end{aligned}
$$

where $\bar{\Omega}_{\gamma}=\left\{x \in R^{n}:\|x\| \leq \gamma\right\}$. By means of Lemma 11, we have

$$
\begin{aligned}
\widehat{f}^{T} & (x) P W_{1} \widehat{g}(x) \\
& \leq \frac{1}{2}\left[\widehat{f}^{T}(x) P W_{1} S^{-1} W_{1}^{T} P \widehat{f}(x)+\widehat{g}^{T}(x) S \widehat{g}(x)\right] \\
& \leq \frac{1}{2} \widehat{f}^{T}(x)\left(P W_{1} S^{-1} W_{1}^{T} P+E^{2} S\right) \widehat{f}(x), \\
\widehat{f}^{T} & (x) P W_{2} \widehat{h}(x) \\
& \leq \frac{1}{2}\left[\widehat{f}^{T}(x) P W_{2} T^{-1} W_{2}^{T} P \widehat{f}(x)+\widehat{h}^{T}(x) T \widehat{h}(x)\right] \\
& \leq \frac{1}{2} \widehat{f}^{T}(x)\left(P W_{2} T^{-1} W_{2}^{T} P+K^{2} T\right) \widehat{f}(x) .
\end{aligned}
$$

By Lemma 12, condition (5) is equivalent to

$$
\begin{aligned}
& P W_{0}+W_{0}^{T} P+P W_{1} S^{-1} W_{1}^{T} P+\sigma P W_{2} T^{-1} W_{2}^{T} P+E^{2} S \\
& \quad+\sigma K^{2} T<0 .
\end{aligned}
$$

From (A.3) and (A.4), it follows that

$$
\begin{aligned}
\widehat{f}(x) & P \mathscr{H}(\lambda, x) \\
= & \widehat{f}^{T}(x) P(C x+\lambda \mathscr{H}(0))-\lambda \widehat{f}(x) P W_{0} \widehat{f}(x) \\
& -\lambda \widehat{f}^{T}(x) P W_{1} \widehat{g}(x)-\lambda \sigma \widehat{f}^{T}(x) P W_{2} \widehat{h}(x) \\
\geq & \widehat{f}^{T}(x) P[C x+\lambda \mathscr{H}(0)] \\
& -\frac{\lambda}{2} \widehat{f}^{T}(x)\left(P W_{0}+W_{0}^{T} P+P W_{1} S^{-1} W_{1}^{T} P\right. \\
\geq & \left.\quad+\sigma P W_{2} T^{-1} W_{2}^{T} P+E^{2} S+\sigma K^{2} T\right) \widehat{f}(x) \\
& \sum_{i=1}^{n}\left[c_{i} p_{i}\left|\widehat{f}_{i}\left(x_{i}\right)\right|\left|x_{i}\right|-\lambda p_{i}\left|\widehat{f}_{i}\left(x_{i}\right)\right|\left|\mathscr{H}(0)_{i}\right|\right] \\
\geq & \sum_{i=1}^{n} c_{i} p_{i}\left|\widehat{f}_{i}\left(x_{i}\right)\right|\left[\left|x_{i}\right|-\frac{\left|\mathscr{H}(0)_{i}\right|}{c_{i}}\right],
\end{aligned}
$$

where $\mathscr{H}(0)_{i}$ denotes the $i$ th element of vector $\mathscr{H}(0)$.

By virtue of Lemma 10, there exist constants $q_{i_{0}}$ and $r_{i_{0}}$ such that

$$
\left|\widehat{f}_{i}\left(x_{i}\right)\right| \geq q_{i_{0}} r_{i_{0}}^{\alpha}, \quad \forall\left|x_{i}\right| \geq r_{i_{0}}, i=1, \ldots, n
$$

Let $a=\max _{1 \leq i \leq n}\left(\left|\mathscr{H}(0)_{i}\right| / c_{i}\right), \mathcal{N}_{k}=\left\{n_{1}, \ldots, n_{k}\right\} \subset\{1, \ldots, n\}$, $\Omega_{\mathscr{N}_{k}}=\left\{x:\left|x_{i}\right| \leq a, i \in \mathcal{N}_{k}, x \in R^{k}\right\}$, for all $k<n$. Define

$$
\widehat{f}_{\mathcal{N}_{k}}(x)=\sum_{i \in \mathscr{N}_{k}} c_{i} p_{i}\left|\widehat{f}_{i}\left(x_{i}\right)\right|\left[\left|x_{i}\right|-a\right] .
$$

Noting that $\Omega_{\mathcal{N}_{k}}$ is a compact subset of $R^{k}$ and $\widehat{f}_{\mathcal{N}_{k}}$ is continuous on $\Omega_{\mathcal{N}_{k}}, \widehat{f}_{\mathscr{N}_{k}}$ can reach its minimum $\min _{x \in \Omega_{\mathcal{N}_{k}}} \widehat{f}_{\mathcal{N}_{k}}(x)$ on $\Omega_{\mathscr{N}_{k}}$.

Let $r_{0}=\max _{1 \leq i \leq n} r_{i_{0}}, l=\min _{1 \leq i \leq n}\left\{c_{i} p_{i} q_{i_{o}} r_{i_{0}}^{\alpha}\right\}, \mathscr{M}_{\mathcal{N}_{k}}=$ $\min _{x \in \Omega_{\mathscr{N}_{k}}} \widehat{f}_{\mathcal{N}_{k}}(x)$, and $\mathscr{M}=\min \left\{\mathscr{M}_{\mathcal{N}_{k}}: \mathscr{N}_{k} \subset\{1, \ldots, n\}\right\}$. Set $\gamma>\max \left\{\sqrt{n}(a-\mathscr{M} / l), \sqrt{n} r_{0}\right\}$ and $x \in \partial \bar{\Omega}_{\gamma}$; then there exist two index sets $\mathcal{N}$ and $\overline{\mathcal{N}}$ such that

$$
\left|x_{i}\right| \leq a, \quad i \in \mathcal{N}, \quad\left|x_{i}\right| \geq a, \quad i \in \overline{\mathcal{N}}
$$

where $\mathcal{N} \cup \overline{\mathcal{N}}=\{1, \ldots, n\}$. Furthermore, there exists an index $\bar{i}_{0}$ in $\overline{\mathcal{N}}$ such that

$$
\left|x_{i_{0}}\right| \geq \frac{\gamma}{\sqrt{n}} \geq \max \left\{a, r_{0}\right\}
$$


By (A.5) and (A.7), for any $x \in \partial \bar{\Omega}_{\gamma}$; and $\lambda \in[0,1]$,

$$
\begin{aligned}
\widehat{f}(x) P \mathscr{H}(\lambda, x) \geq & \sum_{i \in \overline{\mathcal{N}}} c_{i} p_{i}\left|\widehat{f}_{i}\left(x_{i}\right)\right|\left[\left|x_{i}\right|-a\right] \\
& +\sum_{i \in \mathcal{N}} c_{i} p_{i}\left|\widehat{f}_{i}\left(x_{i}\right)\right|\left[\left|x_{i}\right|-a\right] \\
\geq & c_{\bar{i}_{0}} p_{\bar{i}_{0}} \widehat{f}_{\bar{i}_{0}}\left(x_{\bar{i}_{0}}\right)\left[\left|x_{\bar{i}_{0}}\right|-a\right]+\mathscr{M} \\
\geq & c_{\overline{i_{0}}} p_{\bar{i}_{0}} q_{\bar{i}_{0_{0}}} r_{\bar{i}_{0_{0}}}^{\alpha}\left[\left|x_{\overline{i_{0}}}\right|-a\right]+\mathscr{M} \\
\geq & c_{\bar{i}_{0}} p_{\bar{i}_{0}} q_{\bar{i}_{0_{0}}} r_{\bar{i}_{0_{0}}}^{\alpha}\left[\left|x_{\overline{i_{0}}}\right|-a+\frac{\mathscr{M}}{l}\right] \\
\geq & c_{\overline{i_{0}}} p_{\bar{i}_{0}} q_{\bar{i}_{0_{0}}} r_{\bar{i}_{0_{0}}}^{\alpha}\left[\frac{\gamma}{\sqrt{n}}-a+\frac{\mathscr{M}}{l}\right]>0 .
\end{aligned}
$$

This implies that $\mathscr{H}(\lambda, x) \neq 0$ for any $x \in \partial \Omega_{R}$ and $\lambda \in[0,1]$. By applying Lemma $8(1)$,

$$
\operatorname{deg}\left(\mathscr{H}(0, x), \Omega_{R}, 0\right)=\operatorname{deg}\left(\mathscr{H}(1, x), \Omega_{R}, 0\right) .
$$

That is, $\operatorname{deg}\left(\mathscr{H}(x), \Omega_{R}, 0\right)=\operatorname{deg}\left(C x, \Omega_{R}, 0\right)=\operatorname{sgn}|C| \neq 0$, where $|C|$ is the determinant of $C$. By applying Lemma $8(2)$, $\mathscr{H}(x)=0$ has at least a solution in $\Omega_{R}$; that is, the system (4) has at least an equilibrium point.

Let $x_{1}^{*}$ and $x_{2}^{*}$ be two different equilibrium points of the system (4); then

$$
\begin{aligned}
C\left(x_{1}^{*}-x_{2}^{*}\right)= & W_{0}\left(f\left(x_{1}^{*}\right)-f\left(x_{2}^{*}\right)\right)+W_{1}\left(g\left(x_{1}^{*}\right)-g\left(x_{2}^{*}\right)\right) \\
& +\sigma W_{2}\left(h\left(x_{1}^{*}\right)-h\left(x_{2}^{*}\right)\right) .
\end{aligned}
$$

By means of (A.3) and (A.4), we can get

$$
\begin{aligned}
0< & \left(f\left(x_{1}^{*}\right)-f\left(x_{2}^{*}\right)\right)^{T} P C\left(x_{1}^{*}-x_{2}^{*}\right) \\
= & \left(f\left(x_{1}^{*}\right)-f\left(x_{2}^{*}\right)\right)^{T} P W_{0}\left(f\left(x_{1}^{*}\right)-f\left(x_{2}^{*}\right)\right) \\
& +\left(f\left(x_{1}^{*}\right)-f\left(x_{2}^{*}\right)\right)^{T} P W_{1}\left(g\left(x_{1}^{*}\right)-g\left(x_{2}^{*}\right)\right) \\
& +\sigma\left(f\left(x_{1}^{*}\right)-f\left(x_{2}^{*}\right)\right)^{T} P W_{2}\left(h\left(x_{1}^{*}\right)-h\left(x_{2}^{*}\right)\right) \\
\leq & \frac{1}{2}\left(f\left(x_{1}^{*}\right)-f\left(x_{2}^{*}\right)\right)^{T} \\
& \times\left(P W_{0}+W_{0}^{T} P+P W_{1} S^{-1} W_{1}^{T} P\right. \\
& \left.\quad+P W_{2} \sigma T W_{2}^{-1} P+E^{2} S+K^{2} T\right) \\
& \times\left(f\left(x_{1}^{*}\right)-f\left(x_{2}^{*}\right)\right)<0 .
\end{aligned}
$$

This is a contradiction. This shows that the equilibrium point of the system (4) is unique. This completes the proof.

\section{Conflict of Interests}

The authors declare that there is no conflict of interests regarding the publication of this paper.

\section{Acknowledgments}

This work was supported by the National Science and Technology Major Project of China (no. 2011ZX05020-006) and Natural Science Foundation of Hebei Province of China (A2011203103).

\section{References}

[1] J. J. Hopfield, "Neural networks and physical systems with emergent collective computational abilities," Proceedings of the National Academy of Sciences of the United States of America, vol. 79, no. 8, pp. 2554-2558, 1982.

[2] J. J. Hopfield, "Neurons with graded response have collective computational properties like those of two-state neurons," Proceedings of the National Academy of Sciences of the United States of America, vol. 81, no. 10 I, pp. 3088-3092, 1984.

[3] G. Joya, M. A. Atencia, and F. Sandoval, "Hopfield neural networks for optimization: study of the different dynamics," Neurocomputing, vol. 43, pp. 219-237, 2002.

[4] N. Kumaresan and P. Balasubramaniam, "Optimal control for stochastic nonlinear singular system using neural networks," Computers and Mathematics with Applications, vol. 56, no. 9, pp. 2145-2154, 2008.

[5] S. S. Young, P. D. Scott, and N. M. Nasrabadi, "Object recognition using multilayer Hopfield neural network," IEEE Transactions on Image Processing, vol. 6, no. 3, pp. 357-372, 1997.

[6] T. Roska and L. O. Chua, "Cellular neural networks with non-linear and delay-type template elements and non-uniform grids," International Journal of Circuit Theory and Applications, vol. 20, no. 5, pp. 469-481, 1992.

[7] M. Forti and P. Nistri, "Global convergence of neural networks with discontinuous neuron activations," IEEE Transactions on Circuits and Systems - I, vol. 50, no. 11, pp. 1421-1435, 2003.

[8] M. Forti, P. Nistri, and D. Papini, "Global exponential stability and global convergence in finite time of delayed neural networks with infinite gain," IEEE Transactions on Neural Networks, vol. 16, no. 6, pp. 1449-1463, 2005.

[9] M. Forti, M. Grazzini, P. Nistri, and L. Pancioni, "Generalized Lyapunov approach for convergence of neural networks with discontinuous or non-Lipschitz activations," Physica D, vol. 214, no. 1, pp. 88-99, 2006.

[10] G. T. Stamov and I. M. Stamova, "Almost periodic solutions for impulsive neural networks with delay," Applied Mathematical Modelling, vol. 31, no. 7, pp. 1263-1270, 2007.

[11] R. Samli and S. Arik, "New results for global stability of a class of neutral-type neural systems with time delays," Applied Mathematics and Computation, vol. 210, no. 2, pp. 564-570, 2009.

[12] H. Zhang, Z. Wang, and D. Liu, "Global asymptotic stability and robust stability of a class of Cohen-Grossberg neural networks with mixed delays," IEEE Transactions on Circuits and Systems I, vol. 56, no. 3, pp. 616-629, 2009.

[13] Z. Wang, H. Zhang, and W. Yu, "Robust stability criteria for interval Cohen-Grossberg neural networks with time varying delay," Neurocomputing, vol. 72, no. 4-6, pp. 1105-1110, 2009.

[14] H. Wu, "Global exponential stability of Hopfield neural networks with delays and inverse Lipschitz neuron activations," Nonlinear Analysis: Real World Applications, vol. 10, no. 4, pp. 2297-2306, 2009. 
[15] Y. Li and H. Wu, "Global stability analysis in Cohen-Grossberg neural networks with delays and inverse Hölder neuron activation functions," Information Sciences, vol. 180, no. 20, pp. 40224030, 2010.

[16] H. Wu and X. Xue, "Stability analysis for neural networks with inverse Lipschitzian neuron activations and impulses," Applied Mathematical Modelling, vol. 32, no. 11, pp. 2347-2359, 2008.

[17] H. Wu, G. Xu, C. Wu, N. Li, K. Wang, and Q. Guo, "Stability in switched Cohen-Grossberg neural networks with mixed time delays and non-Lipschitz activation functions," Discrete Dynamics in Nature and Society, vol. 2012, Article ID 435402, 22 pages, 2012.

[18] X. Nie and J. Cao, "Stability analysis for the generalized CohenGrossberg neural networks with inverse Lipschitz neuron activations," Computers \& Mathematics with Applications, vol. 57, no. 9, pp. 1522-1536, 2009.

[19] C.-D. Zheng, C.-K. Gong, and Z. Wang, "Stability criteria for Cohen-Grossberg neural networks with mixed delays and inverse Lipschitz neuron activations," Journal of the Franklin Institute, vol. 349, no. 9, pp. 2903-2924, 2012.

[20] S. Haykin, Neural Networks, Prentice-Hall, 1994.

[21] S. Blythe, X. Mao, and X. Liao, "Stability of stochastic delay neural networks," Journal of the Franklin Institute, vol. 338, no. 4, pp. 481-495, 2001.

[22] X. X. Liao and X. Mao, "Exponential stability and instability of stochastic neural networks," Stochastic Analysis and Applications, vol. 14, no. 2, pp. 165-185, 1996.

[23] Z. Wang, Y. Liu, K. Fraser, and X. Liu, "Stochastic stability of uncertain Hopfield neural networks with discrete and distributed delays," Physics Letters A, vol. 354, no. 4, pp. 288-297, 2006.

[24] Y. Chen, A. Xue, X. Zhao, and S. Zhou, "Improved delaydependent stability analysis for uncertain stochastic Hopfield neural networks with time-varying delays," IET Control Theory and Applications, vol. 3, no. 1, pp. 88-97, 2009.

[25] R. Yang, H. Gao, and P. Shi, "Novel robust stability criteria for stochastic Hopfield neural networks with time delays," IEEE Transactions on Systems, Man, and Cybernetics B, vol. 39, no. 2, pp. 467-474, 2009.

[26] Z. Wang, Y. Liu, M. Li, and X. Liu, "Stability analysis for stochastic Cohen-Grossberg neural networks with mixed time delays," IEEE Transactions on Neural Networks, vol. 17, no. 3, pp. 814-820, 2006.

[27] X. Li, "Global robust stability for stochastic interval neural networks with continuously distributed delays of neutral type," Applied Mathematics and Computation, vol. 215, no. 12, pp. 4370-4384, 2010.

[28] W. Su and Y. Chen, "Global robust exponential stability analysis for stochastic interval neural networks with time-varying delays," Communications in Nonlinear Science and Numerical Simulation, vol. 14, no. 5, pp. 2293-2300, 2009.

[29] R. Yang, Z. Zhang, and P. Shi, "Exponential stability on stochastic neural networks with discrete interval and distributed delays," IEEE Transactions on Neural Networks, vol. 21, no. 1, pp. 169-175, 2010.

[30] Q. Zhou and L. Wan, "Exponential stability of stochastic delayed Hopfield neural networks," Applied Mathematics and Computation, vol. 199, no. 1, pp. 84-89, 2008.

[31] T. Huang and C. Li, "Robust exponential stability of uncertain delayed neural networks with stochastic perturbation and impulse effects," IEEE Transactions on Neural Networks and Learning Systems, vol. 23, pp. 867-875, 2012.
[32] X. Lou and B. Cui, "Stochastic exponential stability for Markovian jumping BAM neural networks with time-varying delays," IEEE Transactions on Systems, Man, and Cybernetics B, vol. 37, no. 3, pp. 713-719, 2007.

[33] L. Sheng and H. Yang, "Robust stability of uncertain Markovian jumping Cohen-Grossberg neural networks with mixed timevarying delays," Chaos, Solitons and Fractals, vol. 42, no. 4, pp. 2120-2128, 2009.

[34] J. Yu and G. Sun, "Robust stabilization of stochastic Markovian jumping dynamical networks with mixed delays," Neurocomputing, vol. 86, pp. 107-115, 2012.

[35] Z. Wang, Y. Liu, and X. Liu, "State estimation for jumping recurrent neural networks with discrete and distributed delays," Neural Networks, vol. 22, no. 1, pp. 41-48, 2009.

[36] P. Balasubramaniam, S. Lakshmanan, and S. Jeeva Sathya Theesar, "State estimation for Markovian jumping recurrent neural networks with interval time-varying delays," Nonlinear Dynamics, vol. 60, no. 4, pp. 661-675, 2010.

[37] X. Yang and J. Cao, "Synchronization of Markovian coupled neural networks with nonidentical node-delays and random coupling strengths," IEEE Transactions on Neural Networks, vol. 23, pp. 60-71, 2012.

[38] H. Zhang and Y. Wang, "Stability analysis of Markovian jumping stochastic Cohen-Grossberg neural networks with mixed time delays," IEEE Transactions on Neural Networks, vol. 19, no. 2, pp. 366-370, 2008.

[39] Q. Zhu and J. Cao, "Exponential stability of stochastic neural networks with both Markovian jump parameters and mixed time delays," IEEE Transactions on Systems, Man, and Cybernetics B, vol. 41, no. 2, pp. 341-353, 2011.

[40] Q. Zhu and J. Cao, "Robust exponential stability of markovian jump impulsive stochastic Cohen-Grossberg neural networks with mixed time delays," IEEE Transactions on Neural Networks, vol. 21, no. 8, pp. 1314-1325, 2010.

[41] Q. Zhu, C. Huang, and X. Yang, "Exponential stability for stochastic jumping BAM neural networks with time-varying and distributed delays," Nonlinear Analysis: Hybrid Systems, vol. 5, no. 1, pp. 52-77, 2011.

[42] P. Balasubramaniam, R. Krishnasamy, and R. Rakkiyappan, "Delay-interval-dependent robust stability results for uncertain stochastic systems with Markovian jumping parameters," Nonlinear Analysis: Hybrid Systems, vol. 5, no. 4, pp. 681-691, 2011.

[43] K. Deimling, Nonlinear Functional Analysis, Springer, Berlin, Germany, 1985. 


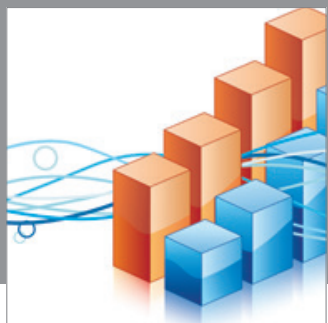

Advances in

Operations Research

mansans

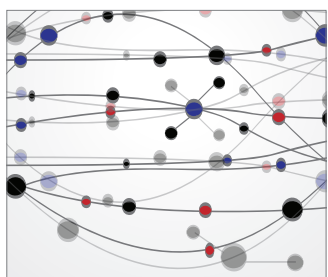

The Scientific World Journal
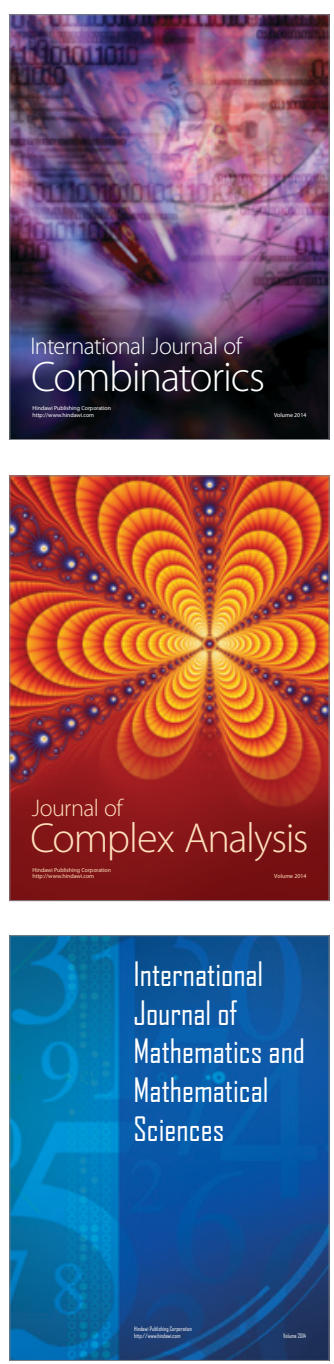
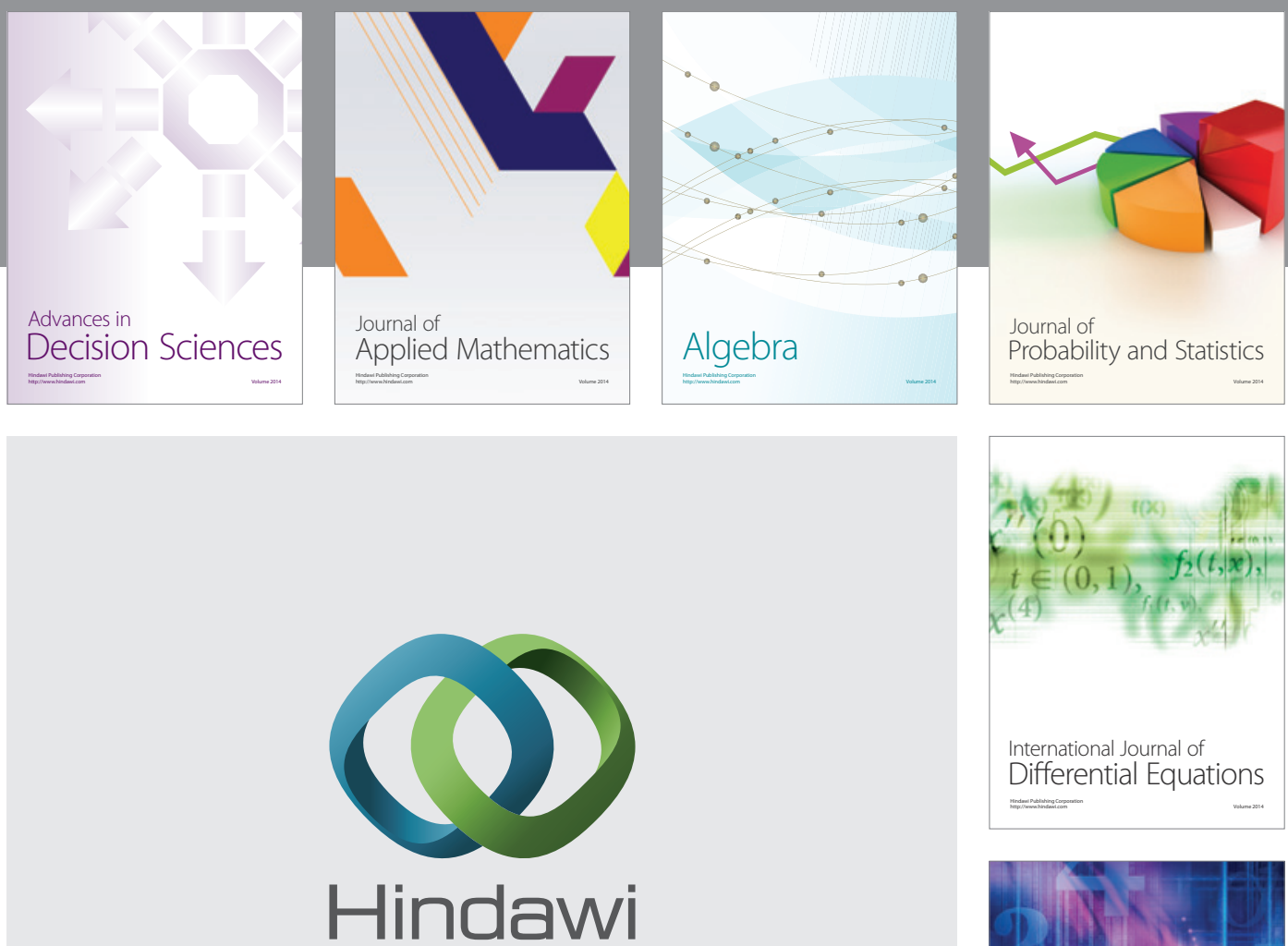

Submit your manuscripts at http://www.hindawi.com
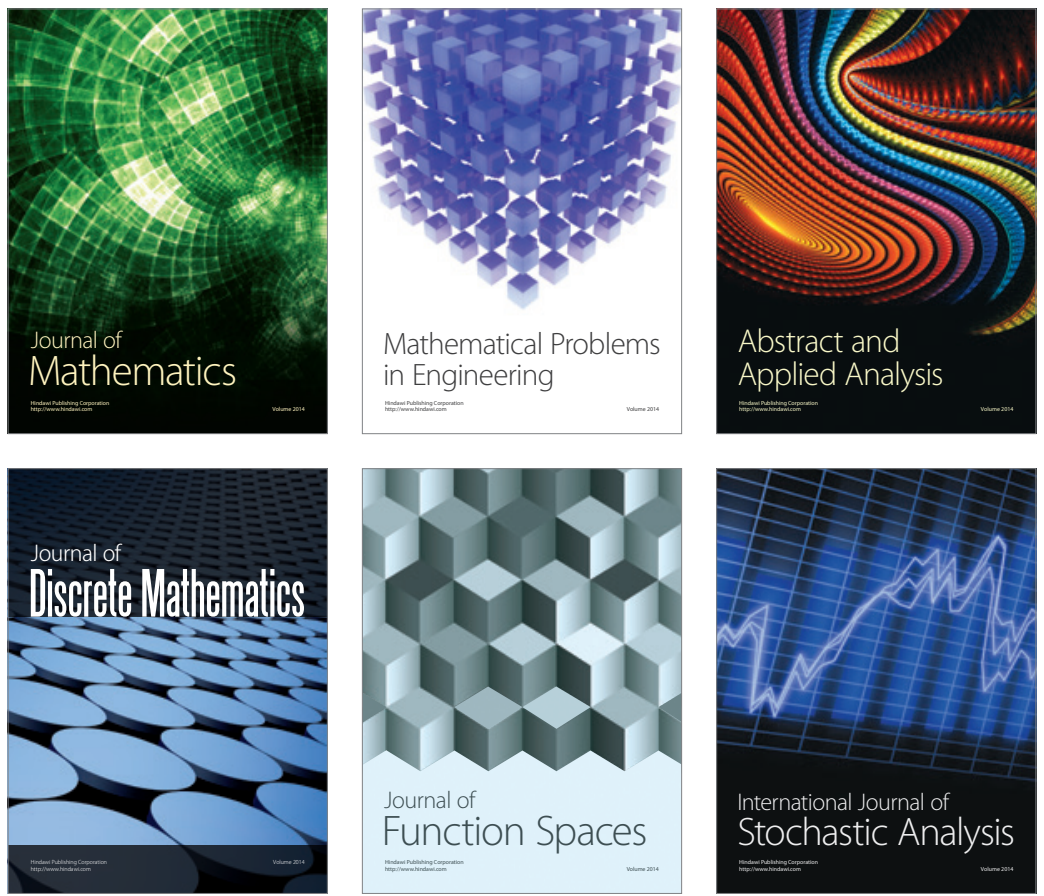

Journal of

Function Spaces

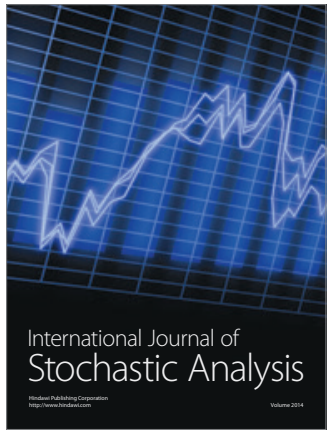

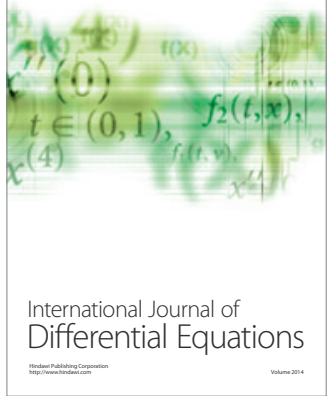
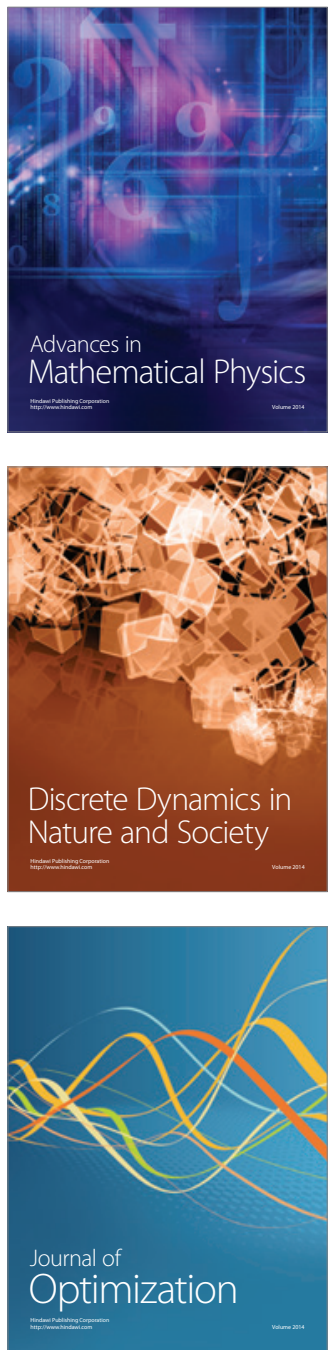